TRANSACTIONS OF THE

AMERICAN MATHEMATICAL SOCIETY

Volume 354, Number 1, Pages 335-362

S 0002-9947(01)02840-9

Article electronically published on June 27, 2001

\title{
SUBGROUP PROPERTIES OF FULLY RESIDUALLY FREE GROUPS
}

\author{
ILYA KAPOVICH
}

\begin{abstract}
We prove that fully residually free groups have the Howson property, that is the intersection of any two finitely generated subgroups in such a group is again finitely generated. We also establish some commensurability properties for finitely generated fully residually free groups which are similar to those of free groups. Finally we prove that for a finitely generated fully residually free group the membership problem is solvable with respect to any finitely generated subgroup.
\end{abstract}

\section{INTRODUCTION}

A group $G$ is called residually free if for any element $g \in G, g \neq 1$ there exists a homomorphism $\phi: G \longrightarrow F$ onto a free group $F$ such that $\phi(g) \neq 1$. Similarly, a group $G$ is called fully residually free (or sometimes $\omega$-residually free) if for any finite collection of nontrivial elements $g_{1} \in G-\{1\}, \ldots, g_{n} \in G-\{1\}$ there exists a homomorphism $\phi: G \longrightarrow F$ onto a free group $F$ such that $\phi\left(g_{1}\right) \neq 1, \ldots, \phi\left(g_{n}\right) \neq 1$. Fully residually free groups have been the subject of extensive and deep research for at least three decades. They are important for a number of reasons.

First, residually free groups are intimately connected to the study of the firstorder logic of free groups and equations over free groups. For example, it is known that fully residually free groups are universally free [58], 21], that is they satisfy all the first-order sentences, containing only universal quantifiers, which hold in all free groups.

Recently remarkable progress has been achieved in understanding the first-order theory of free groups, where understanding the structure of residually free groups played a central part. E.Rips has suggested a new "algebro-geometric" approach to the subject. This approach is being developed by Z.Sela [59]-64 as well as by O.Kharlampovich and A.Myasnikov [35]-[42]. In particular O.Kharlampovich and A.Myasnikov have announced the positive solution to the celebrated Tarski conjecture [36] (see also [42]). A solution to a part of the Tarski conjecture was also recently put forward by Z.Sela [64].

Residually free groups are also closely connected to the theory of the so-called $e x$ ponential groups, which originated in the early sixties with the work of R.Lyndon [47] and G.Baumslag [5].

It also turns out that residually free groups are relevant to the study of groups acting freely on $\Lambda$-trees.

Received by the editors August 26, 1999 and, in revised form, February 8, 2001.

2000 Mathematics Subject Classification. Primary 20F65. 
Many constructions and ideas developed to understand residually free groups and equations over free groups have found applications in other areas, such as the theory of word-hyperbolic groups.

Among the recent remarkable results on the subject is the theorem proved by O.Kharlampovich and A.Myasnikov [38] and Z.Sela [59] which states that finitely generated fully residually free groups are finitely presentable.

In fact O.Kharlampovich and A.Myasnikov 38 proved an even stronger statement, which implies the above result. Before formulating their theorem, let $F$ denote a free group of rank two and let $F^{\mathbb{Z}[x]}$ denote the free $\mathbb{Z}[x]$-tensor completion of $F$.

Theorem 1.1. 38, Let $G$ be a finitely generated group. Then the following hold:

1. The group $G$ is fully residually free if and only if $G$ embeds in $F^{\mathbb{Z}[x]}$.

2. The group $G$ is residually free if and only if $G$ embeds into a finite direct power of $F^{\mathbb{Z}[x]}$

$$
G \leq\left(F^{\mathbb{Z}[x]}\right)^{n}=F^{\mathbb{Z}[x]} \times \cdots \times F^{\mathbb{Z}[x]}
$$

for some integer $n \geq 1$.

For this reason the study of the subgroup structure for the free exponential group $F^{\mathbb{Z}[x]}$ becomes particularly important.

In this paper we prove that the group $F^{\mathbb{Z}[x]}$ shares several important properties with free groups. Before stating our results recall that a group $G$ is said to have the Howson property or to be a Howson group if the intersection of any two finitely generated subgroups of $G$ is again finitely generated.

The main results of this paper are:

Theorem A (cf. Theorem 6.3). The group $F^{\mathbb{Z}[x]}$ has the Howson property.

Theorem B. Let $G$ be a non-abelian finitely generated subgroup of $F^{\mathbb{Z}[x]}$. Suppose $H$ is a finitely generated subgroup of $G$ such that $H$ contains a nontrivial subgroup $N$ which is normal in $G$. Then $H$ has finite index in $G$.

Theorem C. Let $H$ and $K$ be finitely generated subgroups of $F^{\mathbb{Z}[x]}$ such that $H \cap K$ has finite index in both $H$ and $K$. Then $H \cap K$ has finite index in the subgroup generated by $H \cup K$.

All three of these results are well-known for free groups. Applying Theorem 1.1 we immediately obtain the following:

Corollary 1.2. Let $G$ be a fully residually free group. Then the following hold:

1. The group $G$ has the Howson property.

2. Let $H$ and $K$ be finitely generated subgroups of $G$ such that $H \cap K$ has finite index in both $H$ and $K$. Then $H \cap K$ has finite index in the subgroup generated by $H \cup K$.

3. If $G$ is finitely generated and nonabelian and if a finitely generated subgroup $H$ of $G$ contains a nontrivial normal subgroup of $G$, then $H$ has finite index in $G$.

Note that the conclusions of Corollary 1.2 do not necessarily hold if $G$ is just residually free. For instance $G=F(x, y) \times \mathbb{Z}$ is residually free but not fully residually free. It is well-known that such $G$ does not have the Howson property. Also the 
subgroup $F(x, y)$ is finitely generated and normal in $G$ but it has infinite index in $G$.

Finally we study the membership problem for $F^{\mathbb{Z}[x]}$ and show that it is solvable.

Theorem D. Let $G$ be a finitely generated subgroup of $F^{\mathbb{Z}[x]}$.

Then for any finitely generated subgroup $H \leq G$ the group $G$ has solvable membership problem with respect to $H$.

Again, by Theorem 1.1 this immediately implies

Corollary 1.3. Let $G$ be a finitely generated fully residually free group. Then for any finitely generated subgroup $H \leq G$ the group $G$ has solvable membership problem with respect to $H$.

Note that the conclusion of Corollary 1.3 fails for finitely generated residually free groups. For example it is well-known that the group $F_{2} \times F_{2}$ possesses a finitely generated subgroup $H$ such that the membership problem with respect to $H$ is unsolvable [50].

Although the group $F^{\mathbb{Z}[x]}$ is the subject of most of our results, its definition and properties are not relevant for this paper. For the background information on exponential groups and their properties we refer the reader to [53] and [54]. In Section 2 we will give an explicit group theoretic description of the group $F^{\mathbb{Z}[x]}$ and its subgroups. These structural results are then used to show that any finitely generated subgroup $G$ of $F^{\mathbb{Z}[x]}$ can be constructed in two steps. First there is a "core" subgroup $A$ which is torsion-free word-hyperbolic and locally quasiconvex. The group $G$ is then obtained from $A$ by taking a "tree" product of $A$ with several free abelian groups of finite rank. The conclusions of Theorems A, B, C and D are known for locally quasiconvex torsion-free word-hyperbolic groups [43]. The rest of the paper is devoted to proving that these properties are preserved by the kind of "tree" product which leads from $A$ to $G$. Here we follow closely the earlier work of D.Cohen [14], [15]. However, perhaps the most ingenious part of the paper is the proof of Proposition 3.10 which allows us to conclude that the subgroup $A$ is not just word-hyperbolic but locally quasiconvex. We are also able to show in Theorem 3.12 that if $G$ is a fundamental group of a finite graph of groups with cyclic edge groups and torsion-free locally-quasiconvex word-hyperbolic vertex groups, then $G$ is itself locally quasiconvex provided it is word-hyperbolic. This is a new result of considerable independent interest as it expands significantly the important class of locally quasiconvex word-hyperbolic groups.

\section{Structure of FUlly RESiduAlly Free Groups}

The notion of an exponential group was first introduced by R.Lyndon [47] (in connection with the study of equations in free groups) and G.Baumslag [5], [4] (when studying division groups). Roughly speaking, if $R$ is a commutative ring with unit (or just an abelian group with a marked infinite cyclic subgroup), then an $R$-group $G$ is a group with some exponentiation-like "action" of $R$, which satisfies some natural axioms. It turns out that any group $G$ maps to its $R$-completion $G^{R}$, which can be defined in categorical terms and looks much like the group having the same group presentation as $G$, but in the category of $R$-groups rather than in the category of all groups. Moreover, if $G$ is a free group, then its $R$-completion $G^{R}$ turns out to be free in the category of $R$-groups. Of some particular importance 
are the cases when $R$ is the polynomial ring with one variable $\mathbb{Z}[x]$ or when $R$ is the additive group of rational numbers $\mathbb{Q}$.

A detailed and careful discussion about exponential groups and their properties can be found in [53], [54]. Some recent interesting results on the subject are obtained in 20, 34, 32] and other sources.

Definition 2.1. A subgroup $H$ of a group $G$ is said to be malnormal in $G$ if for any $g \in G-H$

$$
g^{-1} H g \cap H=1 .
$$

Two subgroups $K, L$ of $G$ are said to be conjugacy separated in $G$ if for any $g \in G$

$$
g^{-1} K g \cap L=1 .
$$

We recall an explicit description of the group $F^{\mathbb{Z}[x]}$, obtained by A.Myasnikov and V.Remeslennikov in [54].

Proposition 2.2. 54 Let $G=F^{\mathbb{Z}[x]}$ where $F$ is a free group of rank two. Then there exists an infinite increasing chain of subgroups

$$
G_{0}=F \leq G_{1} \leq G_{2} \leq \ldots
$$

such that

1. $G=\bigcup_{i=0}^{\infty} G_{i}$.

2. For every $i \geq 0$ we have $G_{i+1}=G_{i} *_{C} A$ where $C$ is a maximal abelian subgroup of $G_{i}$, which is also malnormal in $G_{i}$ and is a free abelian group of finite rank, and where $A=C \times B$ is a free abelian group of finite rank.

This result gives a certain description of finitely generated subgroups of $F^{\mathbb{Z}[x]}$ using Bass-Serre theory of graphs of groups. However, it will be necessary for us to use a more precise statement describing finitely generated subgroups of $F^{\mathbb{Z}[x]}$ than the one provided by Proposition 2.2.

Namely, we need the result obtained by O.Kharlampovich and A.Myasnikov in [51. Before formulating their theorem we need a few definitions.

The following important notion was first introduced in [27].

Definition 2.3. We will say that an amalgamated free product $G=A *_{C} B$ is separated if $C$ is an abelian subgroup which is malnormal in at least one of $A, B$.

We will say that an HNN-extension $G=\left\langle H, t \mid t^{-1} C_{1} t=C_{2}\right\rangle$ is separated if $C_{1}$, $C_{2}$ are conjugacy separated abelian subgroups of $H$ such that at least one of $C_{1}, C_{2}$ is malnormal in $H$.

Definition 2.4. Let $\mathcal{K}$ be a class of groups.

We will say that a finitely generated group $G$ is constructible over $\mathcal{K}$ if $G$ can be obtained from several finitely generated free groups by taking finitely many separated amalgamated free products and separated HNN-extensions. To be more precise, $G$ is constructible over $\mathcal{K}$ provided there exist finitely many sets $S_{0}, \ldots, S_{n}$ such that

1. For each $i S_{i}$ consists of a finite number of finitely generated subgroups of $G$.

2. $S_{0}$ consists of finitely many groups from $\mathcal{K}$.

3. $S_{n}=\{G\}$. 
4. For every $H \in S_{i}, i \geq 0, H$ is obtained either as a separated amalgamated free products of two groups from $S_{0} \cup \cdots \cup S_{i-1}$ or as a separated HNN-extension of a group from $S_{0} \cup \cdots \cup S_{i-1}$.

We will say that a finitely generated group $G$ is constructible over free groups if $G$ is constructible over the class of finitely generated free groups.

The following is a re-formulation of a result from [51].

Proposition 2.5. Let $G$ be a finitely generated subgroup of $F^{\mathbb{Z}[x]}$ where $F$ is a free group of rank two. Then there exists a subgroup $A$ of $G$ such that the following hold:

1. The group $A$ is constructible over free groups.

2. There exist several maximal cyclic subgroups $U_{1}, \ldots, U_{n}$ of $A$ which are malnormal in $A$ and conjugacy separated in $A$ such that

$$
G=\left(\ldots\left(A *_{U_{1}} B_{1} *_{U_{2}} B_{2}\right) \ldots\right) *_{U_{n}} B_{n}
$$

where $n \geq 1$ and for each $i B_{i}=U_{i} \times C_{i}$ is a free abelian group of finite rank.

Note that in an earlier paper [19] a similar and more precise statement was proved for three-generated fully residually free groups, which therefore applies to all three generator subgroups of $F^{\mathbb{Z}[x]}$.

\section{LOCAL QUASICONVEXITY OF CONSTRUCTIBLE GROUPS}

The goal of this section is to prove that finitely generated groups constructible over free groups are word-hyperbolic and locally quasiconvex.

We first recall some basic definitions regarding word-hyperbolic groups. The background information on the subject can be found in [25], 26], [17], [1, [57] and other sources.

Definition 3.1. Let $(X, d)$ be a metric space. For any point $x, y, z \in X$ we define

$$
(y, z)_{x}=\frac{1}{2}(d(y, x)+d(z, x)-d(y, z)) .
$$

Note that we always have $d(x, y)=(y, z)_{x}+(x, z)_{y}, d(y, z)=(x, z)_{y}+(x, y)_{z}$ and $d(x, z)=(x, y)_{z}+(y, z)_{x}$.

Definition 3.2. Let $\delta>0$ and let $(X, d)$ be a metric space. A triangle with vertices $A, B, C$ and geodesic sides $[A, B],[B, C],[C, A]$ in $(X, d)$ is said to be $\delta$-thin if the following holds.

Let $a \in[B, C], b \in[C, A], c \in[B, A]$ be the points such that $d(B, a)=d(B, c)=$ $(C, A)_{B}, d(A, b)=d(A, c)=(B, C)_{a}$ and $d(C, a)=d(C, b)=(A, B)_{C}$ (see Figure 1 .

Then for any points $b^{\prime} \in[A, C], c^{\prime} \in[A, B]$ with $d\left(A, b^{\prime}\right)=d\left(A, c^{\prime}\right) \leq(B, C)_{A}$ we have

$$
d\left(b^{\prime}, c^{\prime}\right) \leq \delta
$$

and the symmetric condition holds for the vertices $B, C$ of the triangle.

Definition 3.3. A finitely generated group $G$ is said to be word-hyperbolic if for any finite generating set $X$ of $G$ there exists a number $\delta \geq 0$ such that all geodesic triangles in the Cayley graph $\Gamma(G, X)$ are $\delta$-thin. 


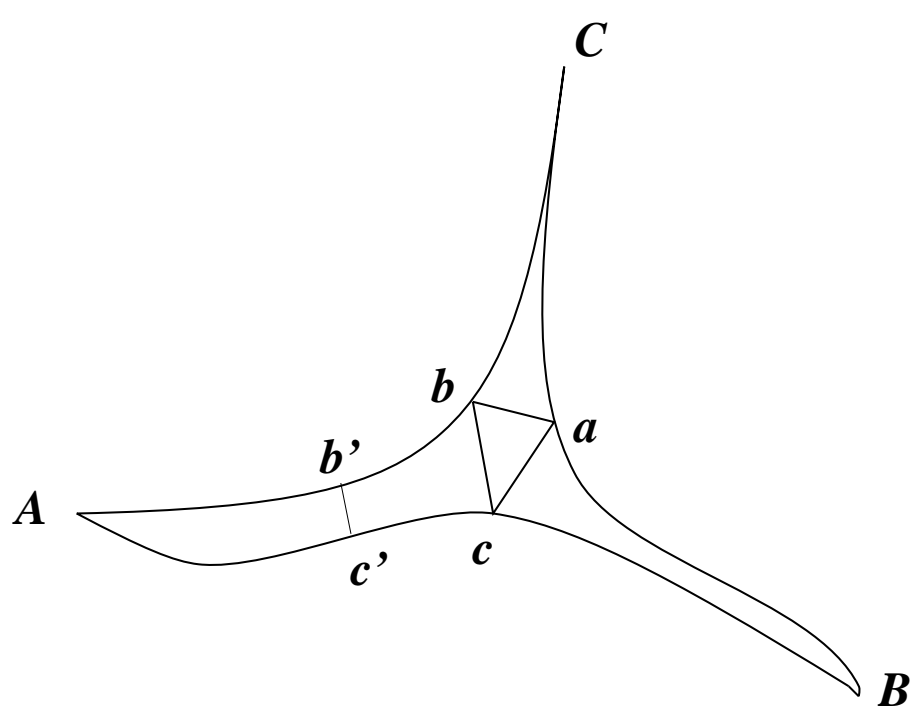

Figure 1. Thin triangle

Definition 3.4. Let $G$ be a word-hyperbolic group. A subgroup $H$ of $G$ is said to be quasiconvex in $G$ if there is a finite generating set $X$ of $G$ and a number $\epsilon \geq 0$ such that the following holds.

For any geodesic $\left[h_{1}, h_{2}\right]$ in $\Gamma(G, X)$ with endpoints $h_{1}, h_{2} \in H$ and for any point $p \in\left[h_{1}, h_{2}\right]$ there exists $h \in H$ such that

$$
d_{X}(p, h) \leq \epsilon
$$

(that is $\left[h_{1}, h_{2}\right]$ is contained in the $\epsilon$-neighborhood $N_{\epsilon}(H)$ of $H$ in the Cayley graph $\Gamma(G, X))$.

Definition 3.5. A word-hyperbolic group $G$ is called locally quasiconvex if every finitely generated subgroup of $G$ is quasiconvex in $G$.

We refer the reader to [43], [55], 28], [24], 66], [67] for more information on quasiconvex subgroups of hyperbolic groups. It is well known, for instance, that a quasiconvex subgroup of a word-hyperbolic group is finitely generated, finitely presentable and itself word-hyperbolic and that the intersection of two quasiconvex subgroups is again quasiconvex, and so finitely generated. This implies the following obvious but important statement.

Proposition 3.6. 66] Every locally quasiconvex word-hyperbolic group $G$ has the Howson property.

Free groups of finite rank are known to be locally quasiconvex [66]. Our goal in this section is to show that groups constructible over free groups are also locally quasiconvex.

It is already known that a separated amalgamated free product of two torsionfree locally quasiconvex word-hyperbolic groups is again locally quasiconvex [24], [32]. 
Proposition 3.7. Let $G=A *_{C} B$ be a separated amalgamated free product where $A, B$ are torsion-free locally quasiconvex word-hyperbolic groups. Then $G$ is also a torsion-free locally quasiconvex word-hyperbolic group.

Proof. By assumption $C$ is malnormal in at least one of $A, B$, say in $A$. Since $C$ is an abelian subgroup of a torsion-free word-hyperbolic group $A$, then $C$ is either trivial or infinite cyclic. Since cyclic subgroups are always quasiconvex in word-hyperbolic groups, this means that $C$ is quasiconvex in both $A$ and $B$ and malnormal in $A$. Hence by the Combination Theorem [12, 23], 35] the group $G=A *_{C} B$ is torsion-free word-hyperbolic. It follows from the main results of [24] and 32 that $G$ is in fact locally quasiconvex.

The case of separated HNN-extensions is more difficult and requires special treatment. Before considering it, let us recall the following fact which is a restatement of the main result in 24]

Proposition 3.8. 24 Let $G=A *_{C} B$ where $A, B$ are torsion-free word-hyperbolic groups, $C$ is quasiconvex in both $A$ and $B$ and $C$ is malnormal in $A$. Let $H$ be a finitely generated subgroup of $G$ such that $g^{-1} \mathrm{Hg} \cap A$ is quasiconvex in $A$ and $g^{-1} \mathrm{Hg} \cap \mathrm{B}$ is quasiconvex in $B$ for any $g \in G$.

Then $G$ is torsion-free word-hyperbolic and $H$ is quasiconvex in $G$.

We will also need the following technical lemma.

Lemma 3.9. Let

$$
G=\left\langle L s \mid s^{-1} p s=q\right\rangle
$$

where $\langle p\rangle,\langle q\rangle$ are malnormal infinite cyclic subgroups in $L$ which are conjugacy separated in $L$. Suppose further that $K=\langle p, q\rangle \leq L$ is a free group of rank two.

Then

1. $\langle s, p\rangle \cap L=K$.

2. The group $\langle s, p\rangle$ is a free group of rank two.

3. The group $G$ can be written as an amalgamated free product

$$
G=L *_{p=p, q=s^{-1} p s}\langle s, p\rangle=L *_{K}\langle s, p\rangle .
$$

Proof. It is easy to show (see, for example, [45]) that $\langle s, p\rangle \cap L=K$.

We will prove that the group $Q=\langle s, p\rangle=\langle s, p, q\rangle$ has the following presentation

$$
Q=\left\langle s, p, q \mid s^{-1} p s=q\right\rangle \text {. }
$$

This obviously implies that $Q$ is a free group of rank two with basis $s, p$.

Claim. Suppose $w$ is a word in $s, p, q$ such that $w=1$ in $Q$. We will show that $w=1$ follows from the relation $s^{-1} p s=q$ by induction on the length of $w$.

If $|w|=0$ then $w=1$ in $F(s, p, q)$ and there is nothing to prove.

Suppose now that $|w|=n>0$ in $F(s, p, q), w={ }_{Q} 1$ and the Claim has been verified for all shorter words. If $w$ is not freely reduced in $F(s, p, q)$, then the Claim follows by induction. Thus we may assume that $w$ is freely reduced.

Since $w={ }_{G} 1$, Britton's Lemma [48] for the HNN-extension (1) implies that $w$ has a subword $u$ of one of the two forms:

1. $u=t^{-1} v(p, q) t$ where $v(p, q)={ }_{G} p^{n}, n \neq 0$,

2. $u=t v(p, q) t^{-1}$ where $v(p, q)={ }_{G} q^{n}, n \neq 0$. 
We will assume that the former is true and it will be clear that the second case is completely analogous.

Thus $u=t^{-1} v(p, q) t$ where $v(p, q)={ }_{G} p^{n}, n \neq 0$. Since by assumption of Lemma 3.9 the subgroup $\langle p, q\rangle$ is free of rank two with basis $p, q$, this implies that $v(p, q)=p^{n}$ in $F(s, p, q)$. Thus $u=t^{-1} p^{n} t$. Using the relation $t^{-1} p t=q$ we can replace $u$ in $w$ by a shorter word $u^{\prime}=q^{n}$ to get a shorter word $w^{\prime}(s, p, q)$, where $w^{\prime}={ }_{Q} 1$. Since $\left|w^{\prime}\right|<|w|$, the inductive hypothesis applies to $w^{\prime}$. This means that $w=1$ is a corollary of a single relation $t^{-1} p t=q$.

This completes the proof of the Claim and of part 2 of Lemma 3.9

Part 3 of Lemma 3.9 is also an elementary exercise on the use of normal forms in amalgamated free products and HNN-extensions and we leave the details to the reader.

Proposition 3.10. Let $G=\left\langle L, t \mid t^{-1} A t=B\right\rangle$ be a separated HNN-extension of a locally quasiconvex torsion-free word-hyperbolic group $L$. Then $G$ is also a locally quasiconvex torsion-free word-hyperbolic group.

Proof. Recall that $A, B$ are abelian (and therefore cyclic) subgroups of $L$ which are conjugacy separated in $L$ and that at least one of $A, B$ (say $A$ ) is malnormal in $L$. If $A=B=1$ then $G=L *\langle t\rangle$ and Proposition 3.7 applies.

Thus we may assume that $A$ and $B$ are infinite cyclic. Recall that in a torsion-free word-hyperbolic group, malnormal infinite cyclic subgroups are exactly maximal infinite cyclic subgroups. Therefore $G$ has the form

$$
G=\left\langle L, t \mid t^{-1} a t=b^{n}\right\rangle
$$

where $n \geq 1$ and $\langle a\rangle,\langle b\rangle$ are malnormal infinite cyclic subgroups of $L$. We may in fact assume that $n=1$. Indeed, if $n>1$, then

$$
G=\left\langle\left(L *_{a=c^{n}}\langle c\rangle\right)=L_{1}, t \mid t^{-1} c t=b\right\rangle
$$

where $\langle c\rangle$ is an infinite cyclic group.

The group $L_{1}$ is torsion-free locally quasiconvex word-hyperbolic by Proposition 3.7. The subgroups $\langle c\rangle,\langle b\rangle$ are easily seen to be malnormal and conjugacy separated in $L_{1}$.

Thus from now on we will assume that

$$
G=\left\langle L, t \mid t^{-1} a t=b\right\rangle
$$

where $L$ is a torsion-free locally quasiconvex word-hyperbolic group and where $A=\langle a\rangle$ and $B=\langle b\rangle$ are malnormal conjugacy separated infinite cyclic subgroups of $L$.

Consider the group $G^{\prime}=G * F(x, y)$. Then $G^{\prime}$ also is an HNN-extension of the group $L^{\prime}=L * F(x, y)$.

$$
G^{\prime}=\left\langle(L * F(x, y))=L^{\prime}, t \mid t^{-1} a t=b\right\rangle .
$$

Note that the group $L^{\prime}=L * F(x, y)$ is torsion-free locally quasiconvex wordhyperbolic by Proposition [3.7. Moreover $A=\langle a\rangle$ and $B=\langle b\rangle$ are malnormal conjugacy separated infinite cyclic subgroups of $L^{\prime}$. Corollary 0.4 in [31] states that any HNN-extension over infinite cyclic subgroups of a locally quasiconvex torsionfree word-hyperbolic group has the Howson property, provided both the associated infinite cyclic subgroups are malnormal in the base group. The HNN-extension (4) is exactly of this sort and therefore $G^{\prime}$ has the Howson property. 
It is proved in [46] that there exist an element $f \in F(x, y)$ such that the subgroup $K=\left\langle a, f^{-1} b f\right\rangle$ is a free group of rank two which is malnormal and quasiconvex in $L^{\prime}$. If we put $s=t f$, then $G^{\prime}$ also has an HNN-presentation

$$
G^{\prime}=\left\langle L^{\prime}, s \mid s^{-1} a s=f^{-1} b f\right\rangle .
$$

It follows from Lemma 3.9 that $\langle s, a\rangle \cap L^{\prime}=K$ and that $\langle s, a\rangle$ is a free group of rank two with basis $s, a$. We can then write $G^{\prime}$ as an amalgamated free product

$$
G^{\prime}=L^{\prime} *_{a=a, f^{-1} b f=s^{-1} a s}\langle s, a\rangle=L^{\prime} *_{K}\langle s, a\rangle
$$

where $Q=\langle s, a\rangle$ is a free group of rank two and so $Q$ is torsion-free locally quasiconvex word-hyperbolic. Recall that $K$ is a free subgroup which is malnormal and quasiconvex in $L^{\prime}$, where $L^{\prime}$ is a torsion-free locally quasiconvex word-hyperbolic group. Thus Proposition 3.8 applies to the amalgamated free product $G^{\prime}=L^{\prime} *_{K} Q$.

By the Combination Theorem [12] $G^{\prime}=L^{\prime} *_{K} Q$ is torsion-free word-hyperbolic. We claim that in fact $G^{\prime}$ is locally quasiconvex.

Indeed, suppose $H$ is a finitely generated subgroup of $G^{\prime}$ and let $g \in G^{\prime}$. We have already observed above that $G^{\prime}$ has the Howson property. Therefore $g^{-1} H g \cap L^{\prime}$ and $g^{-1} H g \cap Q$ are finitely generated. Since $L^{\prime}$ and $Q$ are locally quasiconvex, $g^{-1} H g \cap L^{\prime}$ is quasiconvex in $L^{\prime}$ and $g^{-1} H g \cap Q$ is quasiconvex in $Q$. As we have seen the subgroup $K$ is malnormal in $L^{\prime}$. Therefore by Proposition 3.8 the group $G^{\prime}$ is torsion-free word-hyperbolic and locally quasiconvex. Since $G$ is a finitely generated subgroup of $G^{\prime}$, this implies that $G$ is also torsion-free word-hyperbolic and locally quasiconvex.

Remark 3.11. The most technical part of the proof of Proposition 3.10 is the existence of a conjugating element $f$ such that the subgroup $\left\langle a, f^{-1} b f\right\rangle$ is malnormal, quasiconvex and free of rank two in $L^{\prime}$. The proof of this statement takes most of the article [46], where it is used for other purposes.

The following statement follows immediately from Proposition 3.7 and Proposition 3.10 .

\section{Theorem 3.12.}

1. A group constructible over the class of torsion-free word-hyperbolic locally quasiconvex groups is itself torsion-free word-hyperbolic locally quasiconvex.

2. A group constructible over free groups is torsion-free word-hyperbolic locally quasiconvex.

3. Let $G$ be the fundamental group of a graph of groups $\mathbb{A}$ where all vertex groups are torsion-free word-hyperbolic and locally quasiconvex and all the edge groups are cyclic (either trivial or infinite cyclic). If $G$ is word-hyperbolic, then $G$ is locally quasiconvex.

Proof. Part 1 follows directly from Proposition 3.7 and Proposition 3.10 Part 1 also implies part 2 since finitely generated free groups are locally quasiconvex.

If $G$ is as in part 3, then $G$ can be obtained from the vertex groups of $\mathbb{A}$ by finitely many amalgamated free products and $\mathrm{HNN}$-extensions, corresponding to the edges of $\mathbb{A}$. It is easy to see that each of these amalgams and HNN-extensions is separated since otherwise $G$ would contain a $\mathbb{Z} \times \mathbb{Z}$ subgroup (see, for instance, 45 for details). That however is impossible since $G$ is assumed to be word-hyperbolic. Thus $G$ is constructible from torsion-free locally quasiconvex word-hyperbolic groups. By part 1 this means that $G$ itself is torsion-free locally quasiconvex word-hyperbolic. 


\section{An exCursion in BAss-SERre theory}

In this section we assume familiarity of the reader with the basics of Bass-Serre theory of graphs of groups and groups acting on simplicial trees. For background information the reader is referred to [65, [2] and [16].

Let $G$ be a group acting without inversions on a non-oriented tree $T$. Fix any vertex in $T$ and declare it a base-point of $T$ denoted $O$. Then every non-oriented edge $e$ of $T$ has a naturally defined initial vertex, namely the endpoint of $e$ which is closer to $O$ then the other endpoint of $T$. We will denote the initial vertex of $e$ by $P(e)$. The other endpoint of $e$ is called the terminal vertex of $e$ and denoted $Q(e)$. Thus we orient every edge in the outward from the $O$ direction.

Associated with this action of $G$ on $T$ is a presentation of $G$ as a fundamental group of a graph of groups $\mathbb{A}=T / / G$. The vertex groups of $\mathbb{A}$ are isomorphic to stabilizers in $G$ of some vertices of $T$ and the edge groups of $\mathbb{A}$ are isomorphic to stabilizers in $G$ of some edges of $T$.

It turns out that the question of whether or not $G$ is finitely generated can be read off from the quotient graph of groups $\mathbb{A}$. Namely, if all the vertex groups of $\mathbb{A}$ are finitely generated and the underlying graph $A$ of $\mathbb{A}$ is finite, then $G$ is finitely generated. In terms of the original action of $G$ on $T$, this means that if there are only finitely many $G$-orbits of edges in $T$ and if every stabilizer of a vertex in $G$ is finitely generated, then $G$ is finitely generated.

In [14] D.Cohen obtained a more precise characterization of finitely generated groups acting on trees. To state it we need the following definition.

Definition 4.1. 14 Let $G$ act on a tree $T$ without inversions. Let $O$ be the base-point of $T$ and let every edge of $T$ be oriented away from $O$, as explained above.

We say that an element $g \in G$ is negative for an edge $e$ of $T$ if $g P(e)=Q(g e)$.

For an edge $f$ of $T$ we say that an orbit $G f$ is reversing if for some (and therefore for any) edge $e \in G e$ there exists an element $g \in G$ negative for $e$.

Proposition 4.2. 14 Let $G$ act on a tree $T$ without inversions. Let $O$ be the base vertex of $T$.

1. If $G$ is finitely generated, then there are only finitely many $G$-reversing orbits. If in addition every edge of $T$ has finitely generated stabilizer in $G$, then every vertex of $T$ also has a finitely generated stabilizer in $G$.

2. If $G$ has only finitely many reversing orbits of edges in $T$ and if every vertex of $T$ has finitely generated stabilizer in $G$, then $G$ is finitely generated.

Definition 4.3 (Star of groups). We will call a finite graph of groups $\mathbb{A}$ a star of groups if the underlying graph of $\mathbb{A}$ is a finite tree with a marked vertex $O$ (called the center vertex) and such that every other vertex is connected by a single edge to the base vertex (see Figure 2) . There are exactly $n$ edges in $\mathbb{Z} A$, where $n \geq 1$. Denote these edges by $e_{1}, \ldots, e_{n}$. Their edge groups are $U_{1}, \ldots, U_{n}$ respectively. The non-center endpoints of $e_{1}, \ldots, e_{n}$ are vertices $O_{1}, \ldots, O_{n}$ which have vertex groups $B_{1}, \ldots, B_{n}$.

The fundamental group of the graph of groups $\mathbb{A}$ is an amalgamated free product

$$
G=\left(\ldots\left(A *_{U_{1}} B_{1} *_{U_{2}} B_{2}\right) * \ldots\right) *_{U_{n}} B_{n}
$$




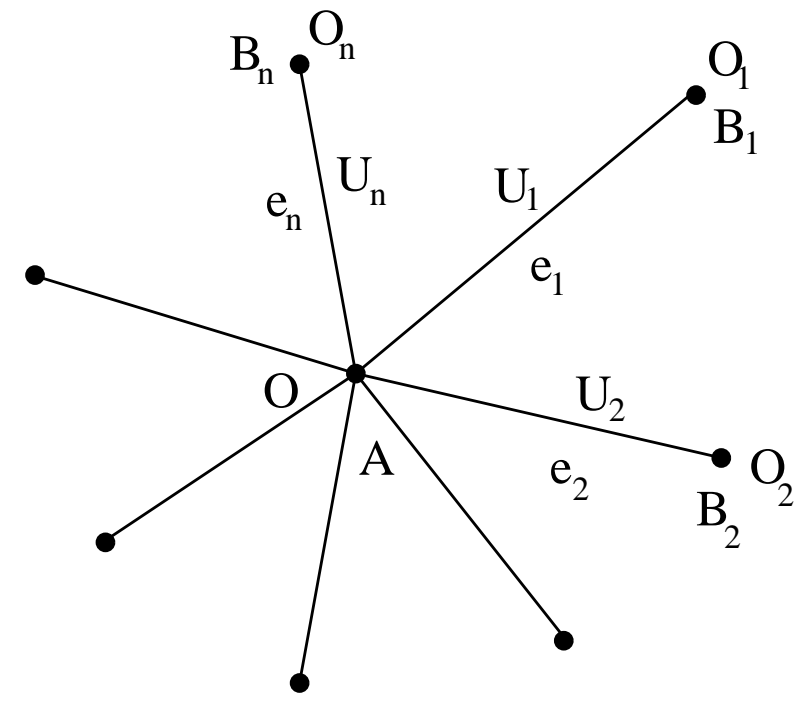

Figure 2. Star of groups

where $n \geq 1$ and $U_{1}, \ldots, U_{n}$ are subgroups of $A$ and where $A$ is the vertex group corresponding to the base vertex of $\mathbb{A}$. In this situation we will also use notation

$$
G=\left(A ; U_{1}, \ldots, U_{n} ; B_{1}, \ldots, B_{n}\right) .
$$

\section{Burns subgroups, amalgams and the Howson property}

The Howson property for amalgamated free products and HNN-extensions was extensively studied in [3, 44, 10], 11, [15], 31] and other sources.

It turns out that the crucial notion in this regard is that of a Burns subgroup, first used by R.Burns [10, [1] (the term "Burns subgroup" was introduced by D.Cohen in [15]).

Definition 5.1. [15] Let $U$ be a subgroup of a group $G$. A subgroup $U$ of a group $G$ is called a Burns subgroup, if $U$ has a left transversal $T$ in $G$ such that $1 \in T$ and the following conditions are satisfied:

1. there is a finite subset $F$ of $U$ such that

$$
U(T-1) \subset T F
$$

2. for any finitely generated subgroup $H$ of $G$ and any $a \in G$ there is a finite subset $F_{1}$ of $U$ such that

$$
a H \subset T F_{1}(H \cap U) .
$$

The following statement is proved by D.Cohen [15] using the basic Bass-Serre theory of graph of groups.

Proposition 5.2. [15] Let $G=A *_{U} B$ where $A, B$ have the Howson property and where $U$ is a Burns subgroup of $A$. Suppose further that for any finitely generated subgroup $H$ of $G$ the intersection $H \cap U$ is finitely generated. Then $G$ has the Howson property. 
We shall need a generalization of this statement for the case of star of groups.

The following definition is a generalization of the notion of a Burns subgroup.

Definition 5.3. We say that the set $U_{1}, \ldots, U_{n}$ is a Burns collection of subgroups of $G$ if the following holds:

1. Each $U_{i}$ is a Burns subgroup of $G$.

2. For each $i \neq j$ we have $U_{i} \cap U_{j}=1$.

3. Let $1 \leq i \leq n$ and let $T$ be the Burns transversal of $U_{i}$ in $G$. Then there exists a finite subset $F_{2}$ of $U_{i}$ such that for any $j, 1 \leq j \leq n$,

$$
U_{j}(T-\{1\}) \subseteq T F_{1} .
$$

Note that if $U$ is a Burns subgroup of $G$, then $\{U\}$ is a Burns collection of subgroups of $G$.

Our goal in this section is to prove the following statement.

Proposition 5.4. Let $A$ be a group with the Howson property. Let $U_{1}, \ldots, U_{n}$ be a Burns collection of subgroups of $A$.

Let $G=\left(A ; U_{1}, \ldots, U_{n} ; B_{1}, \ldots, B_{n}\right)$ where all the $B_{i}$ are Howson groups. Suppose further that $H \cap U_{i}$ is finitely generated for every finitely generated subgroup $H$ of $G$ and for every $1 \leq i \leq n$ (this is the case, for instance, if $U$ is Noetherian, that is, all subgroups of $U$ are finitely generated).

Then $G$ has the Howson property.

The following statement, proved by I.Kapovich [31], allows one to apply the above statements to word-hyperbolic groups.

Proposition 5.5. 31 Let $A$ be a torsion-free word-hyperbolic locally quasiconvex group. Let $U$ be a malnormal infinite cyclic subgroup of $A$. Then $U$ is a Burns subgroup of $A$.

Moreover, any element $t$ of the Burns transversal $T$ of $U$ is shortest (with respect to some fixed word metric on $A)$ in the coset class $t U$.

For the remainder of this section let $G=\left(A ; U_{1}, \ldots, U_{n} ; B_{1}, \ldots, B_{n}\right)$ be an amalgam corresponding to a star of groups $\mathbb{A}$.

Let $T$ be the universal covering Bass-Serre tree corresponding to this graph of groups (see the explicit construction of $T$ in [65], [2]). Then $G$ acts on $T$ without inversion with the quotient graph of groups $\mathbb{A}$.

The underlying graph of $\mathbb{A}$ is a distinguished subset of $T$. We will fix a basevertex $O$ in $T$ to be the center vertex $O$ of $\mathbb{A}$. The stabilizer of $O$ in $G$ is $A$. There are $n$ distinct edges $e_{1}, \ldots, e_{n}$ in $T$ (also coming from the underlying graph of $\mathbb{A}$ ) with initial vertex $O$ and edge stabilizers $U_{1}, \ldots, U_{n}$ respectively. Stabilizers in $G$ of terminal vertices of $e_{1}, \ldots, e_{n}$ are groups $B_{1}, \ldots, B_{n}$ respectively. We denote the terminal vertices of $e_{1}, \ldots, e_{n}$ by $O_{1}, \ldots, O_{n}$, as it was done in $\mathbb{A}$.

There are exactly $n$ distinct $G$-orbits of edges in $T$, represented by edges $e_{1}, \ldots$, $e_{n}$. There are exactly $n+1$ distinct $G$-orbits of vertices in $T$ represented by $O, O_{1}$, $\ldots, O_{n}$. Accordingly we say that a vertex $v$ of $T$ is of $O$-type (respectively $O_{i}$-type) if $v \in G O$ (respectively $v \in G O_{i}$ ). Similarly we say that an edge $e$ of $T$ is of $i$-type if $e \in G e_{i}$.

The following definition is a slight generalization of the notion introduced by D.Cohen in [15].

Definition 5.6. We say that an edge $e$ of $T$ is special if $P(e)$ is of $O$-type but $P(e) \neq O$. 
The following lemma provides a reduction of Proposition 4.2 when one is trying to prove that a subgroup of $G$ is finitely generated.

The proof of the following lemma is an elementary exercise and is identical to the proof of the amalgamated product case in Lemma 3 of [15]. We therefore leave it to the reader.

Lemma 5.7. Let $H$ be a subgroup of $G$. Then there are only finitely many $H$ reversing orbits of edges in $T$ which do not contain a special edge.

The following statement follows immediately from Lemma 2 of [15].

Lemma 5.8. Suppose that $U_{1}, \ldots, U_{n}$ is a Burns collection of subgroups of $A$. Let $U, U^{\prime} \in\left\{U_{1}, \ldots, U_{n}\right\}$. Let $H$ and $K$ be finitely generated subgroups of $A$ and let $a, a^{\prime} \in A$. Then there is a finite subset $F_{3}$ of $U$ such that if $u \in U$ satisfies

$$
a h u=u^{\prime} a^{\prime} k
$$

for some $k \in K, h \in H, u^{\prime} \in U^{\prime}$ with $d k \notin U$, then

$$
u \in(H \cap U) F_{3}(K \cap U) .
$$

We can now prove Proposition 5.4. Our argument closely follows the proof of Theorem 2 of [15], with the difference that Case 2 did not have to be considered in [15].

Proof of Proposition 5.4. Let $K, H$ be finitely generated subgroups of $G$. Let $L=$ $K \cap H$. Since $L$ and $K$ are finitely generated, the assumptions of Proposition 5.4 imply that $g^{-1} L g \cap U_{i}$ and $g^{-1} K g \cap U_{i}$ are finitely generated for each $1 \leq i \leq n$ and for every $g \in G$. Therefore by Proposition $4.2 K \cap G_{v}$ and $L \cap G_{v}$ are finitely generated for any vertex $v$ of $T$. Since all the groups $A, B_{1}, \ldots, B_{n}$ have the Howson property, every vertex group $G_{v}$ also has the Howson property. Hence $L \cap G_{v}=$ $(K \cap H) \cap G_{v}=\left(K \cap G_{v}\right) \cap\left(L \cap G_{v}\right)$ has the Howson property for every vertex $v$ of $T$.

Thus to see that $L$ is finitely generated it is enough, by Proposition 4.2 to show that there are only finitely many $L$-reversing orbits of edges in $T$.

Lemma 5.7 further implies that it is enough to check that there are only finitely many $L$-reversing orbits containing special edges.

Suppose this is not the case. We know by Proposition 4.2 that $H$ and $K$ have only finitely many reversing orbits containing special edges. Therefore there are infinitely many edges $f_{1}, f_{2}, \ldots$ representing distinct reversing $L$-orbits containing a special edge and such that all $f_{1}, f_{2}, \ldots$ lie in the same $H$-orbit and in the same $K$-orbit. Thus there is an edge $f$ such that $H f \cap K f$ contains infinitely many $L$ reversing orbits containing special edges. Without loss of generality we may assume that $f$ is an 1-type edge.

By conjugating the pair $(H, K)$ if necessary we may assume that $f$ is an edge starting at $O$ and, moreover, that $f=e_{1}$. Thus the following condition, which we will refer to as Condition $X$, holds:

Condition $\mathbf{X}$. The set $H e_{1} \cap K e_{1}$ contains infinitely many $L$-reversing orbits containing a special edge.

Recall that $H$ has only finitely many $H$-reversing orbits. Hence for every $i$, $1 \leq i \leq n$, there are finitely many $i$-type edges starting with $O$, say $a_{1} e_{i}, \ldots, a_{s} e_{i}$, where $a_{j} \in A$, such that any $i$-type edge starting at $O$ and lying in an $H$-reversing 


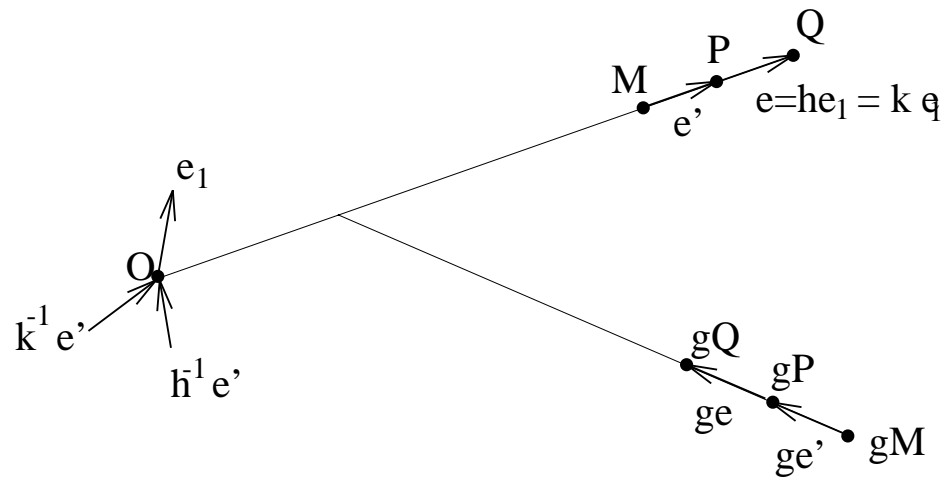

FIGURE 3.

orbit, is $H$-equivalent to one of $a_{j} e_{i}$. That is, if $a e_{i}$ (where $a \in A$ ) belongs to an $H$-reversing orbit, then there is $h \in H$ such that $h a_{j} e_{i}=a e_{i}$ for some $j$. This, in particular, means that $a^{-1} h a_{j}=u \in U_{i}$ and $h=a u a_{j}^{-1} \in A \cap H$. Thus there are only finitely many $(H \cap A)$-orbits of $i$-type edges which start at $O$ and belong to a reversing $H$-orbit. The same argument applies to $K$. Thus, since there are only finitely many types of edges, there is a finite subset $C$ of $A$ such that:

1. For any $i, 1 \leq i \leq n$, every edge in $A e_{i}$, which lies in a reversing $H$-orbit, belongs to $(H \cap A) C e_{i}$;

2. For any $i, 1 \leq i \leq n$, every edge in $A e_{i}$, which lies in a reversing $K$-orbit, belongs to $(\bar{K} \cap \bar{A}) C e_{i}$.

Let $e=h e_{1}=k e_{1} \in H e_{1} \cap K e_{1}$ be a special edge in an $L$-reversing orbit, where $h \in H, k \in K$. Then also we have $k=h u$ for some $u \in U_{1}$.

Put $P=P(e)$ and $Q=Q(e)$. Let $e^{\prime}$ be the edge preceding $e$, so that $P\left(e^{\prime}\right)=M$ and $Q\left(e^{\prime}\right)=P(e)=P$. Suppose $e^{\prime}$ is an $i$-type edge.

If $g \in G$ is negative for $e$ then the terminal vertex of $g e$ is $Q(g e)=g P(e)=g Q\left(e^{\prime}\right)$ which is also the initial vertex of $g e^{\prime}$. Thus $g$ is also negative for $e^{\prime}$ (see Figure 3). Therefore, as the edge $e$, the edge $e^{\prime}$ also belongs to a reversing $L$-orbit.

Consider the edges $h^{-1} e^{\prime}$ and $k^{-1} e^{\prime}$. Since $e=h e_{1}=k e_{1}, P=h O=k O$ and therefore $O=k^{-1} P=h^{-1} P$. Thus both $h^{-1} e^{\prime}$ and $k^{-1} e^{\prime}$ start at $O$ and $h^{-1} e^{\prime} \in A e_{i}, k^{-1} e^{\prime} \in A e_{i}$.

By the choice of the set $C$ above, this means that

$$
h^{-1} e^{\prime}=y c e_{i}, k^{-1} e^{\prime}=z \hat{c} e_{i} \text {, where } c, \hat{c} \in C, y \in H \cap A, z \in K \cap A .
$$

Since $k=h u$ and $k^{-1}=u^{-1} h^{-1}$, this implies that $u z \hat{c} e_{i}=y c e_{i}$, that is $u z \hat{c}=$ ycu' for some $u^{\prime} \in U_{i}$.

There are now two cases to consider.

Case 1. Suppose that $e^{\prime}$ has the same type as $e$, that is $i=1$.

Since $e^{\prime} \neq e, z \hat{c} e_{1}=k^{-1} e^{\prime} \neq k^{-1} e=e_{1}$ and therefore $z \hat{c} \notin U_{1}$.

We can then rewrite the equation $u z \hat{c}=y c u^{\prime}$ as

$$
c^{-1} y^{-1} u=u^{\prime}(\hat{c})^{-1} z^{-1} .
$$

Since $c, \hat{c}$ are in a finite set $C, y^{-1} \in H \cap A, u \in U_{1}, u^{\prime} \in U_{1}, z^{-1} \in K \cap A$ and $(\hat{c})^{-1} z^{-1} \notin U_{1}$, Lemma 5.8 implies that there is a finite subset $S_{1}$ of $U_{1}$, independent 
of the choice of $e \in H e_{1} \cap K$ such that

$$
u \in\left(H \cap U_{1}\right) S_{1}\left(K \cap U_{1}\right) .
$$

Case 2. Suppose that $e^{\prime}$ is of $j$-type for $j \neq 1$.

Subcase 2A. Suppose that $z \hat{c} \notin U_{1}$. Then exactly the same argument as in Case 1 implies that there is a finite subset $S_{2}$ of $U_{1}$, independent of the choice of $e \in H e_{1} \cap K$ such that

$$
u \in\left(H \cap U_{1}\right) S_{2}\left(K \cap U_{1}\right) .
$$

Subcase 2B. Suppose now that $z \hat{c} \in U_{1}$ but $y c \notin U_{1}$. We can then rewrite the equation $u z \hat{c}=y c u^{\prime}$ as

$$
(\hat{c})^{-1} z^{-1} u^{-1}=\left(u^{\prime}\right)^{-1} c^{-1} y^{-1}
$$

where $c^{-1} y^{-1} \notin U_{1}$.

Once again, as in Case 1, Lemma 5.8 implies that there is a finite subset $S_{3}$ of $U_{1}$, independent of the choice of $e \in H e_{1} \cap K$ such that

$$
u^{-1} \in\left(K \cap U_{1}\right) S_{3}\left(H \cap U_{1}\right)
$$

that is

$$
u \in\left(H \cap U_{1}\right)\left(S_{3}\right)^{-1}\left(K \cap U_{1}\right) .
$$

Subcase 2C. Suppose now that $z \hat{c}=u^{\prime} \in U_{1}$ and $y c=u^{\prime \prime} \in U_{1}$.

Recall that $h^{-1} e^{\prime}=y c e_{i}=u^{\prime \prime} e_{i}$ and $k^{-1} e^{\prime}=z \hat{c} e_{i}=u^{\prime} e_{i}$. and therefore $e^{\prime}=$ $h u^{\prime \prime} e_{i}=k u^{\prime} e_{i}$. On the other hand $k=h u$, so that $h u^{\prime \prime} e_{i}=h u u^{\prime} e_{i}$. Hence $u^{\prime \prime} e_{i}=u u^{\prime} e_{i}$ and $\left(u^{\prime \prime}\right)^{-1} u u^{\prime} \in U_{i}$.

However $u, u^{\prime}, u^{\prime \prime} \in U_{1}$ and $U_{1} \cap U_{i}=1$ since $i \neq 1$. Therefore $\left(u^{\prime \prime}\right)^{-1} u u^{\prime}=1$ and

$$
u=u^{\prime \prime}\left(u^{\prime}\right)^{-1}=y c(\hat{c})^{-1} z^{-1} .
$$

Since the subgroup $K \cap A$ is finitely generated, $\hat{c}$ is in a fixed finite set $C$, $z^{-1} \in K \cap A$ and $(\hat{c})^{-1} z^{-1}$ belongs to a Burns subgroup $U_{1}$ of $A$, Definition 5.1 of a Burns subgroup implies that there is a finite subset $S^{\prime}$ of $U$, depending only on $C$ and $K \cap A$, such that

$$
(\hat{c})^{-1} z^{-1} \in S^{\prime}\left(K \cap A \cap U_{1}\right)=S^{\prime}\left(K \cap U_{1}\right) .
$$

Similarly, the subgroup $H \cap A$ is finitely generated, $c^{-1} y^{-1} \in U_{1}$ where $c \in C$ and $y-1 \in H \cap A$. Therefore again Definition 5.1 of a Burns subgroup implies that there is a finite subset $S^{\prime \prime}$ of $U_{1}$, depending only on $C$ and $K \cap A$, such that

$$
c^{-1} y^{-1} \in S^{\prime \prime}\left(K \cap A \cap U_{1}\right)=S^{\prime \prime}\left(K \cap U_{1}\right)
$$

that is,

$$
y c \in\left(K \cap U_{1}\right)\left(S^{\prime \prime}\right)^{-1} \text {. }
$$

Thus

$$
u=y c(\hat{c})^{-1} z^{-1} \in\left(K \cap U_{1}\right)\left(\left(S^{\prime \prime}\right)^{-1} S^{\prime}\right)\left(H \cap U_{1}\right) .
$$

After the analysis of both Case 1 and Case 2 we see that there is a finite subset $S$ of $U_{1}$, independent of the choice of $e \in H e_{1} \cap K$ such that

$$
u \in\left(H \cap U_{1}\right) S\left(K \cap U_{1}\right) \text {. }
$$

We are now in a position to prove that there are only finitely many reversing $L$-orbits in $H e_{1} \cap K e_{1}$, thus obtaining a contradiction with Condition X. 
Indeed, suppose that $e=h e_{1}=k e_{1}$ and $f=h_{1} e_{1}=k_{1} e_{1}$ are edges in $L$ reversing orbits such that $k=h u, k_{1}=h_{1} u_{1}$ and that $u, u_{1}$ correspond to the same $s \in S$ in (7). That is $u=\sigma s \omega$ and $u_{1}=\sigma_{1} s \omega_{1}$ where $s \in S, \sigma, \sigma_{1} \in H \cap U_{1}$ and $\omega, \omega_{1} \in K \cap U_{1}$. Put $h^{\prime}=h \sigma \in H, k^{\prime}=k \omega^{-1} \in K, h_{1}^{\prime}=h_{1} \sigma_{1} \in H$, $k_{1}^{\prime}=k_{1}\left(\omega_{1}\right)^{-1} \in K$. Then we still have $e=k e_{1}=k^{\prime} e_{1}=h e_{1}=h^{\prime} e_{1}$ and $f=k_{1} e_{1}=k_{1}^{\prime} e_{1}=h_{1} e_{1}=h_{1}^{\prime} e_{1}$ since $\sigma, \sigma_{1}, \omega, \omega_{1} \in U_{1}$. Then $k^{\prime}=h^{\prime} s$ and $k_{1}^{\prime}=h_{1}^{\prime} s$ and so

$$
k_{1}^{\prime}\left(k^{\prime}\right)^{-1}=h_{1}^{\prime}\left(h^{\prime}\right)^{-1}=a \in K \cap H=L .
$$

Thus $f=k_{1}^{\prime} e_{1}=a k^{\prime} e_{1}=a e$ with $a \in L=K \cap H$, that is, $e$ and $f$ lie in the same $L$-orbit

Hence there are at most $|S|$ reversing $L$-orbits in $H e_{1} \cap K e_{1}$ which contradicts Condition X.

Remark 5.9. In the proof of Proposition 5.4 we did essentially use the fact that $U_{i} \cap U_{j}=1$ when $i \neq j$. Namely, it was crucial for the analysis of Subcase 2.C. Without this assumption (which is part of the definition of a Burns collection of subgroups) Proposition 5.4 no longer holds. Indeed, suppose that $A=U_{1}=U_{2}=$ $\langle a\rangle$ is an infinite cyclic group and $B_{1}=A \times\langle x\rangle, B_{2}=A \times\langle y\rangle$ are free abelian groups of rank two. Let $G=\left(A ; U_{1}, U_{2} ; B_{1}, B_{2}\right)$. Then it is easy to see that $x, y$ generate a free group in $G$ and that $G=\langle x, y\rangle \times A \cong F_{2} \times \mathbb{Z}$, where $F_{2}$ is a free group of rank two. All the assumptions of Proposition 5.4 are satisfied except the condition $U_{1} \cap U_{2}=1$. However the resulting group $F_{2} \times \mathbb{Z}$ is well known not to be Howson.

\section{Proof of the Howson property For $F_{2}^{\mathbb{Z}[x]}$}

Recall that two paths $\alpha, \beta$ with a common starting point in a metric space $(X, d)$ are said to be $\epsilon$-uniformly close if

- the lengths of $\alpha$ and $\beta$ differ by at most $\epsilon$, and

- for any initial segments $\alpha^{\prime}$ of $\alpha$ and $\beta^{\prime}$ of $\beta$ with the length of $\alpha^{\prime}$ equal the length of $\beta^{\prime}$ we have $d(a, b) \leq \epsilon$ where $a$ and $b$ are the endpoints of $\alpha^{\prime}$ and $\beta^{\prime}$ accordingly.

The definition of a $\delta$-thin triangle can be easily restated in terms of uniformly close paths.

We need the following technical statement to complete the proof of our first main result.

Lemma 6.1. Let $C=\left\langle c_{0}\right\rangle$ and $E=\left\langle e_{0}\right\rangle$ be conjugacy separated infinite cyclic subgroups in a torsion-free word-hyperbolic group $G$. Let $d_{\mathcal{G}}$ be the word metric on $G$ corresponding to a finite generating set $\mathcal{G}$ of $G$.

There exists a constant $N_{0} \geq 0$ with the following properties.

Suppose $w \in G$ is such that $w$ is shortest with respect to the metric $d_{\mathcal{G}}$ in the coset class $w C$. Suppose also that $e \in E$ is an arbitrary element of $E$.

Then for any $y$ shortest in ewC there is $n$ such that $|n| \leq N_{0}$ and $y=e w c_{0}^{n}$.

Proof. Since $G$ is word-hyperbolic, there is $\delta \geq 0$ such that all geodesic triangles in the Cayley graph $\Gamma(G, \mathcal{G})$ of $G$ are $\delta$-thin.

Both $C$ and $E$ are infinite cyclic subgroups of $G$, and therefore they are both quasiconvex in $G$. Hence there is a constant $K^{\prime}>0$ with the following properties: 


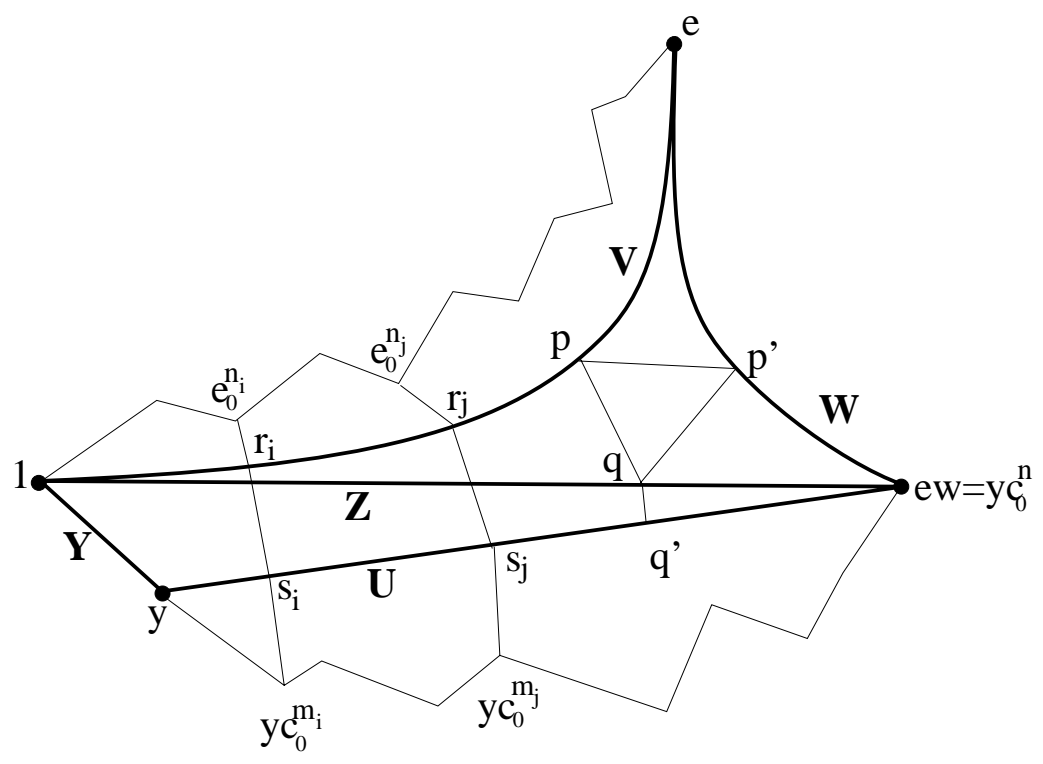

FiguRE 4.

1. If $u$ is a $\mathcal{G}$-geodesic path in $\Gamma(G, \mathcal{G})$ with both endpoints in $C$, then for any point $p$ on $u$ there is $c^{\prime} \in C$ such that $d\left(p, c^{\prime}\right) \leq K^{\prime}$.

2. If $u$ is a $\mathcal{G}$-geodesic path in $\Gamma(G, \mathcal{G})$ with both endpoints in $E$, then for any point $p$ on $u$ there is $e^{\prime} \in C$ such that $d\left(p, e^{\prime}\right) \leq K^{\prime}$.

Let $w$ and $e$ be as in Lemma 6.1. Suppose also that $e w=y c^{n}$ where $y$ is a shortest element in ewC.

Let $W$ and $V$ be $\mathcal{G}$-geodesic representatives of $w$ and $e$ accordingly. Let $Y$ and $U$ be $\mathcal{G}$-geodesic representatives of $y$ and $c_{0}^{n}$ accordingly.

Also let $Z$ be a $\mathcal{G}$-geodesic representative of the element $e w=y c_{0}^{n}$.

Then we have two geodesic triangles in $\Gamma(G, \mathcal{G})$ with sides $Z, V, W$ and $Z, Y, U$ as shown in Figure 4 .

Since the triangle with sides $V, W, Z$ is $\delta$-thin, there are points $p, p^{\prime}$ on $V$ and $P$ and a point $q$ on $Z$ such that

1. the diameter of the set $\left\{p, p^{\prime}, q\right\}$ is at most $\delta$.

2. $d_{\mathcal{G}}(1, q)=d_{\mathcal{G}}(1, p)$ and $d_{\mathcal{G}}\left(p^{\prime}, e w\right)=d_{\mathcal{G}}(q, e w)$.

3. The initial segment $[1, q]$ of $Z$ is $\delta$-uniformly close to the segment $[1, p]$ of $V$.

4. The terminal segment $\left[p^{\prime}, e w\right]$ of $W$ is $\delta$-uniformly close to the terminal segment $[q, e w]$ of $Z$, when they are traveled backwards from ew.

Moreover, since $y$ is shortest in $y C$, by Lemma 4.5 of [13] there exists a constant $K$, depending only on $C$, such that the path $Y U$ and $Z$, when traveled backwards from $y c_{0}^{n}$ to 1 , are $K$-uniformly close in $\Gamma(G, \mathcal{G})$.

In particular this means that for $t=\min \left(d_{\mathcal{G}}\left(p^{\prime}, e w\right), d_{\mathcal{G}}\left(c_{0}^{n}, 1\right)\right)$ the terminal segments of $W$ and $U$ of length $t$ are $\delta+K$-uniformly close, when traveled backwards from $e w$

Since $w$ is shortest in $w C$, Lemma 4.5 of [13] again implies that there is a constant $K_{1}$, depending on $K$ and $C$ such that $t \leq K_{1}$. 
Let $N$ be the number of distinct elements $g \in G$ with $d_{\mathcal{G}}(g, 1) \leq 2 K^{\prime}+K+\delta$.

Put $M=\left(2 K_{1}+1\right)(N+1)\left(2 K^{\prime}+1\right)$.

Suppose that

$$
|U|>M
$$

Since $|U|=d_{\mathcal{G}}\left(c_{0}^{n}, 1\right)>K_{1}$, this means that

$$
d\left(p^{\prime}, e w\right)=\min \left(d_{\mathcal{G}}\left(p^{\prime}, e w\right), d_{\mathcal{G}}\left(c_{0}^{n}, 1\right)\right) \leq K_{1} .
$$

Let $q^{\prime}$ be the point on $U$ with $d_{\mathcal{G}}\left(q^{\prime}, e w\right)=d_{\mathcal{G}}\left(p^{\prime}, e w\right)$ and therefore $d_{\mathcal{G}}\left(p^{\prime}, q^{\prime}\right) \leq$ $K+\delta$. Hence also $d_{\mathcal{G}}\left(q^{\prime}, q\right) \leq K+2 \delta$.

Since we assumed that $|U| \geq\left(2 K_{1}+1\right)(N+1)\left(2 K^{\prime}+1\right)$, we have $d_{\mathcal{G}}\left(y, q^{\prime}\right) \geq$ $\left(2 K_{1}+1\right)(N+1)\left(2 K^{\prime}+1\right)-K_{1} \geq(N+1)\left(2 K^{\prime}+1\right)$. Also for every point $s$ on $Z$ between $y$ and $q^{\prime}$ there is a point $r$ on $V$ between 1 and $p$ such that $d(s, r) \leq K+\delta$. This implies that there are $N+1$ distinct points $s_{1}, s_{2}, \ldots, s_{N+1}$ on $U$ between $y$ and $q^{\prime}$ such that $d\left(s_{i}, s_{j}\right)=\left(2 K^{\prime}+1\right)|i-j|$ for any $1 \leq i, j \leq N+1$.

Recall that since $C$ is quasiconvex, for each $i, 1 \leq i \leq N+1$, there is $c_{0}^{n_{i}}$ such that $d_{\mathcal{G}}\left(s_{i}, y c_{0}^{n_{i}}\right) \leq K^{\prime}$. Note that for $i \neq j$

$$
d_{\mathcal{G}}\left(1, c_{0}^{n_{i}-n_{j}}\right)=d_{\mathcal{G}}\left(y c_{0}^{n_{i}}, y c_{0}^{n_{j}}\right) \geq d_{\mathcal{G}}\left(s_{i}, s_{j}\right)-2 K^{\prime} \geq\left(2 K^{\prime}+1\right)|i-j|-2 K^{\prime}>0 .
$$

Thus $n_{i} \neq n_{j}$ when $i \neq j$.

Also, as was observed above, for each $i$ there is a point $r_{i}$ on $Z$ between 1 and $q$ such that $d_{\mathcal{G}}\left(s_{i}, r_{i}\right) \leq K+\delta$. Since $E$ is quasiconvex, for each $i$ there is $m_{i}$ such that $d_{\mathcal{G}}\left(r_{i}, e_{0}^{m_{i}}\right) \leq K^{\prime}$.

Thus for each $i, 1 \leq i \leq N+1$,

$$
d_{\mathcal{G}}\left(1, e^{-m_{i}} y c_{0}^{n_{i}}\right)=d_{\mathcal{G}}\left(e_{0}^{m_{i}}, y c_{0}^{n_{i}}\right) \leq 2 K^{\prime}+K+\delta .
$$

By the choice of $N$ this means that for some $i \neq j$

$$
e_{0}^{-m_{i}} y c_{0}^{n_{i}}=e_{0}^{-m_{j}} y c_{0}^{n_{j}}=x
$$

Therefore $y=e_{0}^{m_{i}} x c_{0}^{-n_{i}}$ and hence

$$
x=e_{0}^{-m_{j}} y c_{0}^{n_{j}}=e_{0}^{-m_{j}} e_{0}^{m_{i}} x c_{0}^{-n_{i}} c_{0}^{n_{j}}
$$

or

$$
x^{-1} e_{0}^{m_{j}-m_{i}} x=c_{0}^{n_{j}-n_{i}} .
$$

Since $i \neq j$ we have $n_{j}-n_{i} \neq 0$ and therefore $x^{-1} E x \cap C \neq 1$. This contradicts our assumption that $E$ and $C$ are conjugacy separated.

Thus our assumption in (8) that $|U| \geq M$ was false and in fact $|U|=d_{\mathcal{G}}\left(c_{0}^{n}, 1\right)<$ $M$. Therefore there exists a constant $N_{0}$ depending on $M$ and $c_{0}$ such that $|n| \leq N_{0}$. This completes the proof of Lemma 6.1.

Corollary 6.2. Let $A$ be a torsion-free word-hyperbolic group. Let $U_{1}, \ldots, U_{n}$ be infinite cyclic subgroups of $A$ which are malnormal in $A$. Suppose also that $U_{i}$ and $U_{j}$ are conjugacy separated in $A$ for $i \neq j, 1 \leq i, j \leq n$.

Then $U_{1}, \ldots, U_{n}$ is a Burns collection of subgroups of $A$.

Proof. Recall each $U_{i}$ is a Burns subgroup of $A$ by Proposition 5.5 Moreover, by Proposition 5.5 the Burns transversal $T_{i}$ of $U_{i}$ is such that every $t \in T_{i}$ is shortest in the coset class $t U_{i}$. 
Hence by Lemma 6.1 for each $i \neq j$ there is a finite subset $F$ of $U_{i}$ such that

$$
U_{j} T_{i} \subseteq T_{i} F .
$$

Also, since $U_{i}$ itself is a Burns subgroup of $A$ with Burns transversal $T_{i}$, by Definition 5.1 there exists a finite subset $F^{\prime}$ of $U_{i}$ such that

$$
U_{i}\left(T_{i}-\{1\}\right) \subseteq T_{i} F^{\prime}
$$

Since there are only finitely many groups $U_{1}, \ldots, U_{n}$ this implies that all the conditions of Definition 5.3 are satisfied and $U_{1}, \ldots, U_{n}$ is a Burns collection of subgroups of $A$.

We can now prove the first main result of this paper.

Theorem 6.3 (cf. Theorem $\mathrm{A}$ from the Introduction). The group $F_{2}^{\mathbb{Z}[x]}$ has the Howson property.

Proof. Obviously it suffices to prove that a finitely generated subgroup $G$ of $F_{2}^{\mathbb{Z}[x]}$ is Howson.

Then by Proposition $2.5 G$ has a finitely generated subgroup $K$, constructible over free groups, such that either $G=K$ or $K \supsetneqq G$ and

$$
G=\left(K ; C_{1}, \ldots, C_{n} ; B_{1}, \ldots, B_{n}\right)
$$

where

1. $C_{1}, \ldots, C_{n}$ are infinite cyclic subgroups of $K$ which are malnormal in $K$;

2. subgroups $C_{i}$ and $C_{j}$ are conjugacy separated in $K$ whenever $i \neq j$;

3. for each $i B_{i}=C_{i} \times Z_{i}$ where $Z_{i}$ is a nontrivial free abelian group of finite rank.

Note that by Theorem $3.12 K$ is torsion-free, word-hyperbolic and locally quasiconvex. Therefore by Proposition $3.6 \mathrm{~K}$ has the Howson property.

Thus if $G=K$ then $G$ is Howson.

If $G \neq K$, then by Corollary $6.2 C_{1}, \ldots, C_{n}$ is a Burns collection of subgroups of $K$. Therefore by Proposition $5.4 G$ has the Howson property.

\section{Subgroups of Finite indeX}

In this section we obtain some results regarding subgroups of finite index in fully residually free groups. Once again, our arguments will elaborate on those used by D.Cohen in 14$]$ and $[15$.

First we need to develop some more tools regarding groups acting on trees.

Lemma 7.1. Let $G$ be a group acting on a tree $T$ without inversions and let $O$ be the base-vertex of $T$. Let $g \in G$ be a hyperbolic element, that is $g$ fixes no vertex in $T$. Suppose that $g$ is negative for an edge e of $T$.

Then for any $k>0$ the element $g^{k}$ is also negative for $e$.

Proof. Let $L$ be the hyperbolic axis of $g$ in $T$, so that $g$ acts on $L$ by translations of magnitude $\tau>0$.

Let $x$ be the closest vertex of $L$ to $O$, so that $[x, O]$ is the bridge between $L$ and $O$. Let $y$ be the point on $L$ with $d(y, x)=\tau$ and such that $g y=x$.

Then it is clear that $g$ is negative for every edge contained in $S=[y, x] \cup[x, O]$. Moreover, an easy inspection shows that $g$ is not negative for all other edges of $T$. 
$\tau$

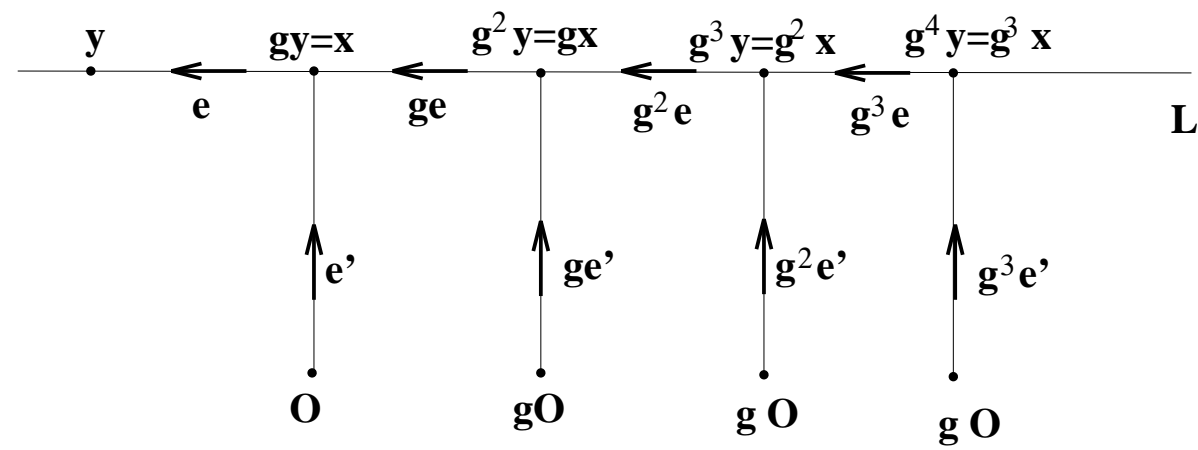

FiguRe 5 .

It is now obvious that for any $k>0 g^{k}$ is negative for any edge contained in $S$ (see Figure 5). This completes the proof of Lemma 7.1

The following statement is due to D.Cohen [14].

Lemma 7.2. [14] Let $G$ be a group acting on a tree $T$ without inversions and let $O$ be the base-vertex of $T$. Let $e$ be an edge of $T$ such that the set

$$
\{g e \mid g \text { is negative for } e\}
$$

is infinite.

Let $h$ be a hyperbolic element of $G$, that is $h$ does not stabilize a vertex in $T$.

Then the set

$\{g e \mid g$ is negative for $e$ and $g$ is conjugate to a power of $h$ in $G\}$

is infinite.

For the rest of this section, unless specified otherwise, let

$$
G=\left(A ; U_{1}, \ldots, U_{n} ; B_{1}, \ldots, B_{n}\right)
$$

be the fundamental group of a star of groups $\mathbb{A}$ (see Figure 2).

Let $T$ be the Bass-Serre covering tree of $\mathbb{A}$ with the standard action of $G$. As usual, we think of the underlying graph of $\mathbb{A}$ as a distinguished part of $T$. Then, as before in Section 5, let the base-vertex $O$ of $T$ be the vertex corresponding to the center of the star $\mathbb{A}$, so that the stabilizer of $O$ in $G$ is $A$. We will use the same convention regarding edges $e_{1}, \ldots, e_{n}$, vertices $O_{1}, \ldots, O_{n}$, regarding $O$-type vertices, $O_{i}$-type vertices and $i$-type edges as in Section 5 ,

The following proposition is similar to Theorem 8 in [14].

Proposition 7.3. Let $H$ be a finitely generated subgroup of $G$ which is not conjugate to a subgroup of a vertex group of $A$.

Suppose also that one of the following holds:

1. the group $H$ contains a subgroup $N$ which is normal in $G$ and such that $N$ is not contained in a conjugate of a vertex group of $A$.

2. for any $g \in G$ and $h \in H$ there exists $k>0$ such that $g^{-1} h^{k} g \in H$. 
Then for any $i=1, \ldots, n$ the double coset index

$$
\left\{H g U_{i} \mid g \in G\right\}
$$

is finite.

Proof. It is easy to see that for any edge $e$ of $T$ the set $\{g e \mid g$ is negative for $e\}$ is infinite.

Then there is $h \in N$ (or just $h \in H$, in case 2) such that $h$ does not fix any vertex of $T$. The same, of course, is true for any nonzero power of $h$.

Let $e$ be an edge of $T$. By Lemma 7.2 the set

$$
\{g e \mid g \text { is negative for } e \text { and } g \text { is conjugate to a power of } h \text { in } G\}
$$

is infinite.

Since, in case $1, g^{-1} h g \in N \leq H$ for any $g \in G$, for this case we have

$$
\{g e \mid g \text { is negative for } e \text { and } g \in H\}
$$

is infinite. In particular, this means that $e$ belongs to an $H$-reversing orbit.

Suppose that (9) does not hold in case 2. Then there is $g \in G$ and $m \neq 0$ such that $g^{-1} h^{m} g \notin H$ but $g^{-1} h^{m} g$ is negative for $e$. By Lemma 7.1 this means that for any $k>0$ the element $g^{-1} h^{m k} g$ is negative for $e$.

By assumption in case 2 there is $m>0$ such that $g^{-1} h^{m k} g \in H$. Thus again $e$ belongs to an $H$-reversing orbit.

We have shown that in both case 1 and case 2 of Proposition 7.3 e belongs to an $H$-reversing orbit. Since $e$ was chosen arbitrarily, this means that every edge of $T$ is contained in an $H$-reversing orbit, that is to say every $H$-orbit of edges is $H$-reversing.

However, $H$ is finitely generated, and so by Proposition 4.2 there are only finitely many $H$-reversing orbits. This means that there are only finitely many $H$-orbits of edges in $T$.

For any $i, 1 \leq i \leq n$, the numbers of $H$-orbits of $i$-type edges is equal to the number of double cosets $\left\{H g U_{i} \mid g \in G\right\}$. This implies the statement of Proposition 7.3

We recall the following statement proved by D.Cohen in [15].

Lemma 7.4. 15] Let $C$ be a Burns subgroup of a group $B$ and let $H$ be a finitely generated subgroup of $B$.

Then for any $b \in B-C$ the intersection of an $(H, C)$-double coset and an $\left(H, b^{-1} C b\right)$-double coset in $B$ contains only finitely many right $H$-cosets $H b^{\prime}$ in $B$.

Lemma 7.5. Suppose that $U_{1} \neq A$ is a Burns subgroup of $A$. Suppose also that $K \cap A$ is finitely generated for any finitely generated subgroup $K$ of $G$.

Then for any $a \in A-U_{1}$ and for any finitely generating subgroup $H$ of $G$ the intersection of an $\left(H, U_{1}\right)$-double coset in $G$ and an $\left(H, a^{-1} U_{1} a\right)$-double coset in $G$ contains only finitely many right $H$-cosets of $G$.

Proof. By conjugating $H$ and $U_{1}$ (and the star of groups presentation of $A$ ) we only need to prove this for $H U \cap H a^{-1} U a$.

Any right $H$-coset in $H U \cap H a^{-1} U a$ is of the form $H u$ where

$$
h u=a^{-1} u^{\prime} a
$$


and where $h \in H, u, u^{\prime} \in U_{1}$. Thus $h \in H \cap A$. The group $H \cap A$ is finitely generated by assumption. Therefore by Lemma 7.4 there are only finitely many possibilities for $H \cap A$-right cosets of $u$ and thus for right $H$-cosets of $u$.

Proposition 7.6. Let $G=\left(A ; U_{1}, \ldots, U_{n} ; B_{1}, \ldots, B_{n}\right)$ where $U_{1}$ is a Burns subgroup of $A$. Assume also that $A \neq U_{1}$ and that $A \cap K$ is finitely generated for any finitely generated subgroup $K$ of $G$.

Let $H$ be a finitely generated subgroup of $G$, such that $H$ is not contained in a conjugate of $A, B_{1}, \ldots$ or $B_{n}$.

Suppose also that $H$ satisfies one of the following:

1. the group $H$ contains a subgroup $N$ which is normal in $G$ and such that $N$ is not contained in a conjugate of a vertex group of $A$.

2. for any $g \in G$, and $h \in H$ there exists $k>0$ such that $g^{-1} h^{k} g \in H$.

Then $H$ has finite index in $G$.

Proof. By Proposition 7.3 there are only finitely many double coset classes $\mathrm{Hg}_{1}$ in $G$.

Let $G=H g_{1} U_{1} \cup \cdots \cup H g_{p} U_{1}$.

Suppose that $H$ has infinite index in $G$. Every $H g_{i} U_{1}$ is closed under $H$ multiplication on the left and is therefore a union of right $H$-cosets $g H$. Since $H$ has infinite index in $G$, at least one of these sets, say $H g_{1} U_{1}$, consists of infinitely many right $H$-cosets.

Let $a \in A$ be such that $a \notin U_{1}$. Then

$$
H g_{1} U_{1} a \subset H g_{1} U_{1} \cup \cdots \cup H g_{p} U_{1} .
$$

Therefore there is some $H g_{j} H$ such that $H g_{1} U_{1} a \cap H g_{j} H$ contains infinitely many right $H$-cosets.

Note that $H g_{1} U_{1} a=H g_{1} a a^{-1} U_{1} a$ is an $\left(H, a^{-1} U_{1} a\right)$-double coset. Hence we obtain a contradiction with the conclusion of Lemma 7.5.

We can now prove the remaining main results of this paper.

Theorem 7.7 (cf. TheoremB from the Introduction). Let $G$ be a finitely generated subgroup of $F_{2}^{\mathbb{Z}[x]}$.

Suppose $H$ is a finitely generated non-abelian subgroup of $G$ which contains a nontrivial normal subgroup $N$ of $G$.

Then $H$ has finite index in $G$.

Proof. Since $G$ is a finitely generated subgroup of $F_{2}^{\mathbb{Z}[x]}$, by Proposition $[2.5 G$ has the form

$$
G=\left(A ; U_{1}, \ldots, U_{n} ; B_{1}, \ldots, B_{n}\right)
$$

where $A$ is constructible over free groups and therefore (by Theorem 3.12) torsionfree word-hyperbolic and locally quasiconvex, where $U_{1}, \ldots, U_{n}$ are infinite malnormal cyclic subgroups of $A$ pairwise conjugacy separated in $A$ and where each $B_{i}=U_{i} \times C_{i}$ is a free abelian group of finite rank.

If $G=A$ then $G$ is torsion-free word-hyperbolic and locally quasiconvex. Therefore the conclusion of Theorem 7.7 holds by Theorem 3 of [43].

Suppose now that $G \neq A$. We may assume that $B_{i} \neq U_{i}$ and $C_{i} \neq 1$ for each $i$. Since $N$ is non-abelian, $G$ is non-abelian. 
If $A$ is abelian, then $A$ is infinite cyclic. Since $U_{1}, \ldots, U_{n}$ are infinite cyclic and pairwise conjugacy separated in $A$, we conclude that $n=1$. Since $U_{1}$ is malnormal in $A$, this means that $A=U_{1}$, and $G=U_{1} \times C_{1}=B_{1}$ is abelian, contrary to our assumptions.

Thus $A$ is non-abelian, and so $A$ is a non-elementary word-hyperbolic group which contains a free subgroup of rank two [26].

Recall that $N \neq 1$ is normal in $G$. In this case it is easy to see that $N$ is not conjugate to a subgroup of $A$ or $B_{i}$.

Indeed, suppose that $N \leq A$ or $N \leq B_{i}$. Since $A \neq U_{i}$ and $B_{i} \neq A_{i}$, the fact that $N$ is normal in $G$ implies $N \leq U_{i}$. Recall that $1 \neq N$ and that $U_{i}$ is a malnormal infinite cyclic subgroup of $A$. Thus $N$ is an infinite normal cyclic subgroup of a torsion-free word-hyperbolic group $A$. This implies that $A$ itself is infinite cyclic, contrary to our earlier conclusion that $A$ is non-elementary.

The subgroup $U_{1}$ is a Burns subgroup of $A$ and $G$ has the Howson property. Therefore by Proposition 7.6 $H$ has finite index in $G$.

Theorem 7.8 (cf. Theorem $\mathrm{C}$ of the Introduction). Let $H, K$ be finitely generated subgroups $F_{2}^{\mathbb{Z}[x]}$. Suppose that $L=H \cap K$ has finite index in both $H$ and $K$.

Then $L$ has finite index in the subgroup $G=\langle H, K\rangle$.

Proof. The group $G$ is a finitely generated subgroup of $F_{2}^{\mathbb{Z}[x]}$.

If $G$ is abelian the statement of Theorem 7.8 obviously holds. Assume now that $G$ is not abelian.

Since $L=H \cap K$ has finite index in both $H$ and $K$ and $G=\langle H, K\rangle$, for any element $g \in G$

$$
L \cap g^{-1} L g \text { has finite index in both } L \text { and } g^{-1} L g .
$$

In particular, this means for any $g \in G$ and any $f \in L$ there is $k>0$ such that $g^{-1} f^{k} g \in L$.

Since $G$ is finitely generated and fully residually free, by Proposition 2.5 it has the form

$$
G=\left(A ; U_{1}, \ldots, U_{n} ; B_{1}, \ldots, B_{n}\right)
$$

where $A$ is constructible over free groups and therefore torsion-free word-hyperbolic and locally quasiconvex (see Theorem 3.12 , where $U_{1}, \ldots, U_{n}$ are infinite malnormal cyclic subgroups of $A$ pairwise conjugacy separated in $A$ and where each $B_{i}=$ $U_{i} \times C_{i}$ is a free abelian group of finite rank.

If $G=A$ then the statement of Theorem 7.8 follows from Theorem 1 of 43 since $A$ is locally quasiconvex and torsion-free.

Suppose $G \neq A$. Since $G$ is non-abelian, we may assume that $A$ is non-abelian as well. Thus $A$ is a non-elementary word-hyperbolic group and it contains a free subgroup of rank two 26. Since $G \neq A$ we may also assume that $C_{i} \neq 1, U_{i} \neq B_{i}$ for each $i$.

Recall that $L$ is a finitely generated subgroup of $G$ such that for any $g \in G$ and any $f \in L$ there is $k>0$ such that $g^{-1} f^{k} g \in L$.

It is not hard to see that in this case this means that $L$ is not conjugate to a subgroup of $A$ or $B_{i}$.

Indeed, suppose that $L$ is a subgroup of $B_{i}$. Recall that $A \neq U_{i}$ and $B_{i} \neq U_{i}$. If there is $f \in L-U_{i} \subseteq B_{i}-U_{i}$, then take an $a \in A-U_{i}$. Since $B_{i}=U_{i} \times U_{i}$ is 
a free abelian group of finite rank, $f^{k} \notin U_{i}$ for any $k>0$. Therefore $a^{-1} f^{k} a \notin B_{i}$ and so $a^{-1} f^{k} a \notin L$, contrary to our assumptions. Thus $L \leq B_{i}$ implies $L \leq U_{i}$.

Suppose that $L \leq A$. If there is $f \in L-U_{i}$, then $f^{k} \notin U_{i}$ for any $k>0$ since $U_{i}$ is malnormal in $A$. Then for any $b \in B_{i}-U_{i}$ and any $k>0, b^{-1} f^{k} b \notin A$ and so $b^{-1} f^{k} b \notin L$, contrary to our assumption. Thus $L \leq A$ implies $L \leq U_{i}$ for each $i$.

Assume now that $L \leq U_{i}$ for some $i$. Since $L \neq 1, L$ is an infinite cyclic subgroup of $A$ such that for any $f \in L$ and any $a \in A-L$ there is $k>0$ such that $a^{-1} f^{k} a \in L$. Since $L$ is infinite cyclic, this means that $a^{-1} L a \cap L$ has finite index in both $L$ and $a^{-1} L a$, that is $a$ belongs to the virtual normalizer $V N_{A}(L)$. Thus $V N_{A}(L)=A$. Infinite cyclic subgroups of word-hyperbolic groups are always quasiconvex [1, so $L$ is quasiconvex in $A$. Since $L$ is quasiconvex in $A$ and infinite, a theorem of [43] implies that $L$ has finite index in $V N_{A}(L)$. But $G$ is torsion-free and $L$ is infinite cyclic. Hence $V N_{A}(L)$ is infinite cyclic itself. But $V N_{A}(L)=A$ which contradicts our assumption that $A$ is non-elementary and contains a non-abelian free subgroup.

Thus $L$ is not conjugate to a subgroup of $A$ or $B_{i}$. Therefore by Proposition 7.6 $L$ has finite index in $G$, as required.

\section{THE MEMBERSHIP PROBLEM}

In this section if $G=\langle X\rangle$ is a group with a generating set $X$, and if $w$ is a word in $X^{ \pm 1}$, we will denote by $\bar{w}$ the element $g \in G$ represented by $w$.

Definition 8.1. Let $G=\langle X\rangle$ be a finitely generated group, where $X$ is a finite set.

Let $H \leq G$ be a subgroup of $G$. We say that $G$ has solvable membership problem with respect to $H$ if there exists an algorithm which, given any word $w$ in $X^{ \pm 1}$, determines whether or not $\bar{w} \in H$.

More generally, we say that $G$ has solvable membership problem if there exists an algorithm which, given any finite set of words $w, w_{1}, \ldots, w_{n}$ in $X^{ \pm 1}$ decides whether or not $\bar{w} \in\left\langle\overline{w_{1}}, \ldots, \overline{w_{1}}\right\rangle$. (It is easy to see that these notions do not depend on the choice of a finite generating set of $G$.)

The membership problem for amalgamated products was originally studied by K.Mihailova [49] and by S.G.Ivanov [29]. The general case of a tree product and the fundamental group of a graph of groups has been considered in a series of papers by V.N.Bezverkhnii [6]- 9 .

The following fact is a corollary of the main result of V.N.Bezverkhnii in [7] (see also [8] for the same statement).

Proposition 8.2. Let $G$ be the fundamental group of a finite graph of groups $\mathbb{A}$ where all edge groups are Noetherian and all vertex groups are finitely generated.

Suppose also that the following conditions are satisfied:

1. Every vertex group $A_{v}$ of $\mathbb{A}$ has solvable membership problem.

2. There is an algorithm which, given a finite subset $Y$ of a vertex group $A_{v}$ and an edge $e$ with initial vertex $\alpha(e)=v$, finds a finite set of generators for the subgroup $\langle Y\rangle \cap \alpha_{e}\left(A_{e}\right)$ of $A_{v}$.

3. There is an algorithm which, given a finite subset $Y$ of a vertex group $A_{v}$, an element $a \in A_{v}$ and an edge $e$ with initial vertex $\alpha(e)=v$, determines if the intersection $a\langle Y\rangle \cap \alpha_{e}\left(A_{e}\right)$ is nonempty.

Then for any finitely generated subgroup $H \leq G$ the group $G$ has solvable membership problem with respect to $H$. 
The conditions of Proposition [8.2, although they seem technical, are easily seen to be satisfied for the star of groups that arises in Proposition [2.5 when one makes use of Theorem 3.12 .

Theorem 8.3 (cf. Theorem $\mathrm{D}$ from the Introduction). Let $G \leq F_{2}^{\mathbb{Z}[x]}$ be a finitely generated group. Then for any finitely generated subgroup $H \leq G$ the group $G$ has solvable membership problem with respect to $H$.

Proof. By Proposition 2.5 and Theorem 3.12 the group $G$ is the fundamental group of a star of groups $G=\left(A ; U_{1}, \ldots, U_{n} ; B_{1}, \ldots, B_{n}\right)$ where $A$ is torsion-free wordhyperbolic and locally quasiconvex, the groups $B_{i}$ are free abelian groups of finite rank, the groups $U_{1}, \ldots, U_{n}$ are malnormal infinite cyclic subgroups of $A$ which are pairwise conjugacy separated in $A$ and each $U_{i}$ is a direct factor in $B_{i}$, that is $B_{i}=U_{i} \times C_{i}$.

We will show now that all the conditions of Proposition 8.2 are satisfied for this graph of groups. The crucial point will be the fact that $A$ is locally quasiconvex.

Conditions (1), (2) and (3) obviously holds for the finitely generated free abelian groups $B_{i}$. Recall that word-hyperbolic groups are biautomatic with the biautomatic structure given by the language of all geodesic words. Quasiconvex subgroups in this situation are exactly those which are rational with respect to this biautomatic structure, that is those whose pre-image in the language of geodesic words is again a regular language. For an overview of the theory of automatic groups the reader is referred to $[18,[13$ and 22 .

Let $S$ be a finite generating set of $A$. Recall that all finitely generated subgroups in $A$ are quasiconvex. Hence, by the result of I.Kapovich [30], there exists an algorithm which, given any finite set of words $Y$ in $S^{ \pm 1}$ finds a finite state automaton $M_{H}$ over the alphabet $S^{ \pm 1}$ such that the language accepted by $M_{H}$ is exactly the set of $S$-geodesic words representing the elements of $H=\langle\bar{Y}\rangle$ (here $H \leq A$ ).

It is also known from the theory of automatic groups that there is an algorithm which, given a word $w$ in $S^{ \pm 1}$ finds a word $v$ in the automatic structure on $A$, that is an $S$-geodesic word $v$, such that $\bar{v}=\bar{w}$ (see Theorem 2.3.10 of [18]). To see if $\bar{w} \in H$, it remains to check whether the word $v$ is accepted by the automaton $M_{H}$. Thus condition (1) of Proposition 8.2 holds for all the vertex groups in the star of groups $G=\left(A ; U_{1}, \ldots, U_{n} ; B_{1}, \ldots, B_{n}\right)$.

Let $U_{i}=\langle u\rangle$ be one of the amalgamated subgroups. Since $U_{i}$ is quasiconvex in $A$, there is a finite state automaton $M_{U_{i}}$ which accept exactly those $S$-geodesic words which represent elements of $U_{i}$.

We can then form a finite state automaton $M^{\prime}$ which accepts $S$-geodesic words representing elements of $H \cap U_{i}$. Using the automaton $M^{\prime}$ it is now easy to find a finite generating set for $H \cap U_{i}$ (see the proof of Theorem 8.3.1 in [18]). Thus condition (2) holds for $A$.

Let $a$ be an arbitrary word in $S^{ \pm 1}$. Using the comparison automata of the biautomatic structure on $A$ and the automaton $M_{H}$ we can find a finite state automaton $M_{a}$ such that the language accepted by $M_{a}$ consists exactly of those $S$-geodesic words which represent elements of $\bar{a} \overline{L\left(M_{H}\right)}=\bar{a} H$ (see the proof of Theorem 2.5.7 in [18]). We can now use $M_{a}$ and $M_{U_{i}}$ to form a finite state automaton $M^{\prime \prime}$ which accepts exactly those $S$-geodesic words which represent elements of $a H \cap U_{i}$. It is now easy to check if $a H \cap U_{i}$ is empty or not by verifying whether the language accepted by $M^{\prime \prime}$ is empty. Thus condition (3) of Proposition 8.2 holds for $A$.

This completes the proof of Theorem 8.3 


\section{REFERENCES}

[1] J.Alonso, T.Brady, D.Cooper, V.Ferlini, M.Lustig, M.Mihalik, M.Shapiro and H.Short. Notes on word hyperbolic groups. In: Group theory from a geometrical viewpoint. Proceedings of the workshop held in Trieste, É. Ghys, A. Haefliger and A. Verjovsky (editors). World Scientific Publishing Co., 1991 MR 93g:57001

[2] H. Bass, Covering theory for graphs of groups, J. Pure Appl. Algebra 89 (1993), no. 1-2, 3-47 MR 94j:20028

[3] B. Baumslag, Intersections of finitely generated subgroups in free products, J. London Math. Soc. 41 (1966), 673-679 MR 33:7396

[4] G. Baumslag, On free D-groups, Comm. Pure Appl. Math. 18 (1965), 25-30 MR 31:2323

[5] G. Baumslag, Some aspects of groups with unique roots, Acta Math. 104 (1960), 217-303 MR 23:A191

[6] V. N. Bezverkhnii, Solvability of the inclusion problem in a class of HNN-groups, (Russian), in "Algorithmic problems of the theory of groups and semigroups", 20-62, Tul'sk. Gos. Ped. Inst., Tula, 1981 MR 84h:20020

[7] V. N. Bezverkhnii, Solution of the occurrence problem in some classes of groups with one defining relation, (Russian), in "Algorithmic problems in the theory of groups and semigroups", 3-21, 126, Tul'sk. Gos. Ped. Inst., Tula, 1986 MR 89e:20065

[8] V. N. Bezverkhnii, Solution of the occurrence problem in a class of groups. I. (Russian), in "Problems in group theory and homological algebra" , 40-53, Matematika, Yaroslav. Gos. Univ., Yaroslavl', 1990 MR 93c:20059

[9] V. N. Bezverkhnii, Solution of the occurrence problem in a certain class of groups. II. (Russian), in "Problems in group theory and homological algebra", 122-142, Yaroslav. Gos. Univ., Yaroslavl', 1991 MR 94a:20054

[10] R. G. Burns, On the finitely generated subgroups of an amalgamated product of two groups, Trans. Amer. Math. Soc. 169 (1972), 293-306 MR 51:8260

[11] R. G. Burns, Finitely generated subgroups of HNN groups, Canad. J. Math. 25 (1973), 1103-1112 MR 48:8642

[12] M. Bestvina and M. Feighn, The combination theorem for negatively curved groups, J. Diff. Geom. 35 (1992), 85-101 MR 93d:53053

[13] G. Baumslag, S. Gersten, M. Shapiro and H. Short, Automatic groups and amalgams, J. Pure and Appl. Algebra 76 (1991), no. 3, 229-316 MR 93a:20048

[14] D. Cohen, Subgroups of HNN groups, J. Austral. Math. Soc. 17 (1974), 394-405 MR 56:8702

[15] D. Cohen, Finitely generated subgroups of amalgamated free products and HNN groups, J. Austral. Math. Soc. Ser. A 22 (1976), no. 3, 274-281 MR 55:12821

[16] D. Cohen, Combinatorial group theory: a topological approach, London Mathematical Society Student Texts, 14; Cambridge University Press, Cambridge, 1989 MR 91d:20001

[17] M. Coornaert, T. Delzant, and A. Papadopoulos, Géométrie et théorie des groupes. Les groupes hyperboliques de Gromov. Lecture Notes in Mathematics, 1441; Springer-Verlag, Berlin, 1990 MR 92f:57003

[18] D. Epstein, J. Cannon, D. Holt, S. Levy, M. Paterson, W. Thurston, Word Processing in Groups, Jones and Bartlett, Boston, 1992 MR 93i:20036

[19] B. Fine, A. Gaglione, A. Myasnikov, G. Rosenberger, and D. Spellman, A classification of fully residually free groups of rank three or less, J. Algebra 200 (1998), no. 2, 571-605 MR 99b:20053

[20] A. Gaglione, A. Myasnikov, V. Remeslennikov, and D. Spellman, Formal power series representations of free exponential groups, Comm. Algebra 25 (1997), no. 2, 631-648 MR 98g:20045

[21] A. Gaglione and D. Spellman, Even more model theory of free groups, in "Infinite groups and group rings (Tuscaloosa, AL, 1992)", 37-40, World Sci. Publishing, River Edge, NJ, 1993 MR 96k:20046

[22] S. Gersten and H. Short, Rational subgroups of biautomatic groups, Ann. Math. (2) 134 (1991), no. 1, 125-158 MR 92g:20092

[23] R. Gitik, On the combination theorem for negatively curved groups, Internat. J. Algebra Comput. 6 (1996), no. 6, 751-760 MR 98b:20058b errata MR 98b:20058c

[24] R. Gitik, On quasiconvex subgroups of negatively curved groups, J. Pure Appl. Algebra 119 (1997), no. 2, 155-169 MR 98h:20057 
[25] M. Gromov, Hyperbolic Groups, in Essays in Group Theory (G.M.Gersten, editor), MSRI Publ. 8, 1987, 75-263 MR 89e:20070

[26] E. Ghys and P. de la Harpe (editors), Sur les groupes hyperboliques d'aprés Mikhael Gromov, Birkhäuser, Progress in Mathematics series, vol. 83, 1990. MR 92f:53050

[27] D. Gildenhuys, O. Kharlampovich, and A. Myasnikov, CSA-groups and separated free constructions, Bull. Austral. Math. Soc. 52 (1995), no. 1, 63-84 MR 96h:20053

[28] R. Gitik, M. Mitra, E. Rips, M. Sageev, Widths of subgroups, Trans. Amer. Math. Soc. 350 (1998), no. 1, 321-329 MR 98e:20048

[29] S. G Ivanov, The membership problem for a free product of groups with an amalgamated subgroup, Sibirsk. Matem. Zhurnal. 16 (1975), no. 6, 1155-1171 MR 54:7639

[30] I. Kapovich, Detecting quasiconvexity: algorithmic aspects, in "Geometric and computational perspectives on infinite groups (Minneapolis, MN and New Brunswick, NJ, 1994)", 91-99, DIMACS Ser. Discrete Math. Theoret. Comput. Sci., 25, Amer. Math. Soc., Providence, RI, 1996 MR 97e:20051

[31] I. Kapovich, Amalgamated products and the Howson property, Canad. Math. Bull. 40 (1997), no. 3, 330-340 MR 98i:20022

[32] I. Kapovich, Quasiconvexity and amalgams, Internat. J. Algebra Comput. 7 (1997), no. 6, 771-811 MR 98k:20057

[33] I. Kapovich, A non-quasiconvexity embedding theorem for word-hyperbolic groups, Math. Proc. Cambridge Phil. Soc. 127 (1999), no. 3, 461-486 MR 2000i:20070

[34] O. Kharlampovich, E. Lioutikova and A. Myasnikov, Equations in the Q-completion of a torsion-free hyperbolic group, Trans. Amer. Math. Soc. 351 (1999), no. 7, 2961-2978 MR 99j:20039b

[35] O. Kharlampovich and A. Myasnikov, Hyperbolic groups and free constructions, Trans. Amer. Math. Soc. 350 (1998), no. 2, 571-613 MR 98d:20041

[36] O. Kharlampovich and A. Myasnikov, Tarski's problem about the elementary theory of free groups has a positive solution, Electron. Res. Announc. Amer. Math. Soc. 4 (1998), 101-108 MR 99k:20051

[37] O. Kharlampovich and A. Myasnikov, Irreducible affine varieties over a free group, I. Irreducibility of quadratic equations and Nullstellensatz, J. Algebra 200 (1998), no. 2, 472-516 MR 2000b:20032a

[38] O. Kharlampovich and A. Myasnikov, Irreducible affine varieties over a free group, II. Systems in triangular quasi-quadratic form and description of residually free groups, J. Algebra 200 (1998), no. 2, 517-570 MR 2000b:20032b

[39] O. Kharlampovich and A. Myasnikov, Description of fully residually free groups and irreducible affine varieties over a free group, Summer School in Group Theory in Banff, 1996, 71-80, CRM Proc. Lecture Notes, 17, Amer. Math. Soc., Providence, RI, 1999 MR 99j:20032

[40] O. Kharlampovich and A. Myasnikov, Implicit function theorem over free groups, preprint, McGill University, 1999; http://www.math.mcgill.ca/olga/publications.html

[41] O. Kharlampovich and A. Myasnikov, Equations over fully residually free groups, preprint, McGill University, 1999; http://www.math.mcgill.ca/olga/publications.html

[42] O. Kharlampovich and A. Myasnikov, Elementary theory of free nonabelian groups, preprint, McGill University, 1999; http://www.math.mcgill.ca/olga/publications.html

[43] I. Kapovich, and H. Short, Greenberg's theorem for quasiconvex subgroups of word hyperbolic groups, Canad. J. Math. 48 (1996), no. 6, 1224-1244 MR 98g:20060

[44] A. Karrass and D. Solitar, The subgroups of a free product of two groups with an amalgamated subgroup, Trans. Amer. Math. Soc. 150 (1970), 227-255

[45] I. Kapovich and R.Weidmann, On the structure of two-generated word-hyperbolic groups, Math. Z. 231 (1999), no. 4, 783-801 MR 2000f:20068

[46] I. Kapovich and D. Wise, The equivalence of some residual properties of word-hyperbolic groups, J. Algebra 223 (2000), no. 2, 562-583 CMP 2000:07

[47] R. Lyndon, Groups with parametric exponents, Trans. Amer. Math. Soc. 96 (1960), 518 533 MR 27:1487

[48] R. Lyndon and P. Schupp. Combinatorial Group Theory. Springer-Verlag, New York, 1977. MR 58:28182

[49] K. A. Mihailova, The occurrence problem for free products of groups. (Russian), Matem. Sbornik 75 (1968), no. 117, 199-210 MR 36:5231 
[50] C. F. Miller III, Decision problems in algebraic classes of groups (a survey), in "Word problems: decision problems and the Burnside problem in group theory (Conf., Univ. California, Irvine, Calif. 1969; dedicated to Hanna Neumann)", pp. 507-523. Studies in Logic and the Foundations of Math., Vol. 71, North-Holland, Amsterdam, 1973 MR 53:3121

[51] A.Myasnikov, Subgroups of free exponential groups, City College of CUNY, preprint, 1999

[52] W. Magnus, A. Karrass and D. Solitar, Combinatorial group theory: Presentations of groups in terms of generators and relations, Interscience Publishers [John Wiley and Sons, Inc.], New York-London-Sydney, 1966 MR 23:A1696

[53] A. Myasnikov and V. Remeslennikov, Degree groups, I. Foundations of the theory and tensor completions. (Russian), Sibirsk. Mat. Zh. 35 (1994), no. 5, 1106-1118 MR 95m:20047

[54] A. Myasnikov and V. Remeslennikov, Exponential groups, II. Extensions of centralizers and tensor completion of CSA-groups, Internat. J. Algebra Comput. 6 (1996), no. 6, 687711 MR 97j:20039

[55] M. Mihalik and W. Towle, Quasiconvex subgroups of negatively curved groups, J. Pure Appl. Algebra 95 (1994), no. 3, 297-301 MR 95h:20045

[56] J. McCammond and D. Wise, Coherence, local quasiconvexity and the perimeter of 2complexes, preprint, 1998

[57] A. Yu. Olshanskii, On residualing homomorphisms and G-subgroups of hyperbolic groups, Intern. J. Alg. Comp. 3 (1993), no. 4, 365-409 MR 94i:20069

[58] V. Remeslennikov, $\exists$-free groups and groups with length function, Second International Conference on Algebra (Barnaul, 1991), 369-376, Contemp. Math., 184, Amer. Math. Soc., Providence, RI, 1995 MR 96d:20020

[59] Z. Sela, Diophantine geometry over groups I: Makanin-Razborov diagrams, Hebrew University, preprint; http://www.ma.huji.ac.il/ zlil/

[60] Z. Sela, Diophantine geometry over groups II: Completions, closures and formal solutions, Hebrew University, preprint; http://www.ma.huji.ac.il/〜zlil/

[61] Z. Sela, Diophantine geometry over groups III: Rigid and solid solutions, Hebrew University, preprint; http://www.ma.huji.ac.il/ ${ }^{\sim}$ zlil/

[62] Z. Sela, Diophantine geometry over groups IV: An iterative procedure for validation of a sentence, Hebrew University, preprint, http://www.ma.huji.ac.il/ zlil/

[63] Z. Sela, Diophantine geometry over groups V: Quantifier elimination, Hebrew University, preprint; http://www.ma.huji.ac.il/ ${ }^{\sim}$ zlil/

[64] Z. Sela, Diophantine geometry over groups VI: The elementary theory of a free group, Hebrew University, preprint; http://www.ma.huji.ac.il/〜zlil/

[65] J.-P. Serre, Trees. Springer-Verlag, Berlin-New York, 1980 MR 82c:20083

[66] H. Short, Quasiconvexity and a theorem of Howson's, in "Group theory from a geometrical viewpoint (Trieste, 1990)", 168-176, World Sci. Publishing, River Edge, NJ, 1991 MR 93d:20071

[67] E. Swenson, Quasi-convex groups of isometries of negatively curved spaces, Topol. Appl. 110 (2001), no. 1, 119-129 CMP 2001:07

Department of Mathematics, University of Illinois at Urbana-Champaign, 1409 West Green Street, Urbana, Illinois 61801

E-mail address: kapovich@math.uiuc.edu 